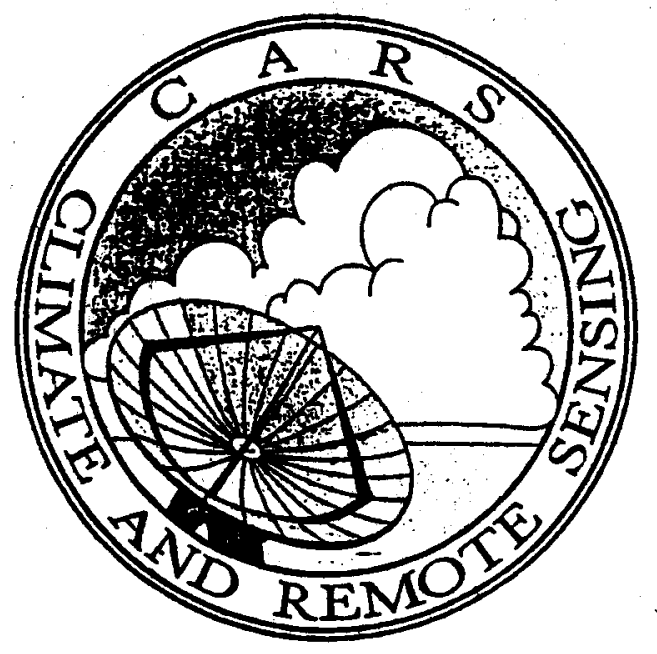

NAGD-697

50250

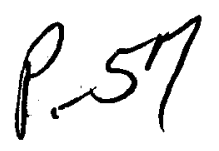

\title{
CALIBRATION OF NOAA-7 AVHRR, GOES-5 AND GOES-6 VISSR/VAS SOLAR CHANNELS
}

\author{
R. FROUIN \\ C. GAUTIER \\ (NASA-CE-180103) CALIERAIICN CF NOAA-7 \\ AVHEF, GOES-5 AN L GCES-6 VISSF, VAS SOLAR \\ N87-16062 \\ CHANNEIS Fial Technical EefCrt, 1 Fet. \\ $1 \subseteq E 5$ - 31 Jan. $19 E 6$ (SCriffs Institution of \\ Cceasography. La Jc11a) $57 \mathrm{p}$ \\ $\operatorname{CSCL} 22 \mathrm{~A} \quad \mathrm{G} 3 / 19$ \\ Unclas \\ 43236 \\ California Space Institute \\ Scripps Institution of Oceanography \\ La Jolla, CA 82093
}

August 1986

CARS Report \#005 


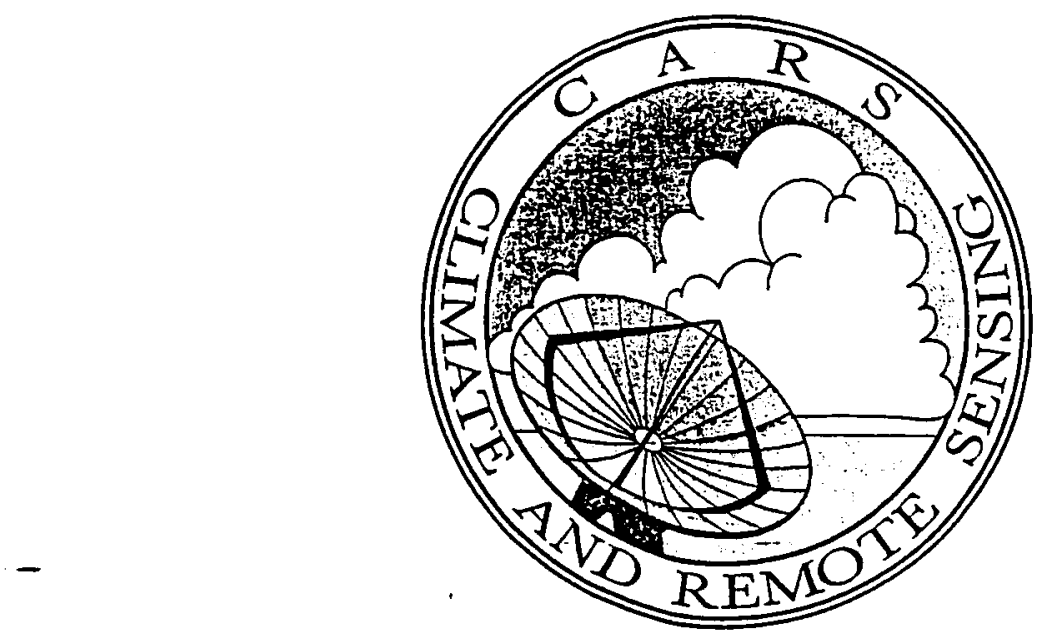

\title{
CALIBRATION OF NOAA-7 AVHRR, GOES-5 AND GOES-6 VISSR/VAS SOLAR CHANNELS
}

\author{
R. FROUIN \\ C. GAUTIER \\ California Space Institute \\ Scripps Institution of Oceanography \\ La Jolla, CA 92093
}

August 1986

CARS Report \#005 


\begin{abstract}
The NOAA-7, GOES-5 and GOES-6 VISSR/VAS solar channels have been calibrated for the periods from October 1983 through January 1985 (NOAA-7, GOES-6) and from October 1983 through July 1984 (GOES-5). The White Sands Monument area in New Mexico, whose relectance properties are well known, and space are used as calibration targets. The shortwave reflected terrestrial irradiance that is measured at satellite altitude is computed using a fairly accurate radiative transfer model which accounts for multiple scattering and bidirectional effects (Tanré et al., 1979). The ground target reflectance and relevant characteristics of the overlying atmosphere are estimated from climatological data (ozone amount, aerosol size-frequency distribution and refractive index) and observations at the nearest meterorological sites (water vapor amount, visibility). The approach is believed to produce accuracies of 8 to $13 \%$ depending on the channel considered. The results obtained for the 15-month period do not indicate any drift in the solar channels of both types of instrument. The gain changes, about $15 \%$ of the mean values, are largely attributed to inhomogeneities of the ground target (shading effects due to the presence of dunes). No systematic effect of the normalization procedure applied by NOAA to the raw VISSR/VAS data is detected. There is some evidence that the GOES-5 solar channels gradually deteriorated from March 1984 until the failure of the satellite in July 1984. Comparisons of gains determined in orbit with those before launch show that the NOAA-7 solar channels read higher by about $15 \%$. The disparities cannot be explained by model errors and must have occurred before the time period analyzed here.
\end{abstract}




\section{Introduction}

The Visible Infrared Spin Scan Radiometer/Vertical Atmospheric Sounder (VISSR/VAS) and the Advanced Very High Resolution Radiometer (AVHRR) aboard the meteorological satellites operated by the National Oceanic and Atmospheric Administration (NOAA) were primarily intended to provide frequent, high resolution, visible and infrared images for monitoring weather events and temperature stratifications over large areas. Sucessfully applied to cloud detection, the data in the visible and near-infrared bands have also proved suitable for other environmental applications, in particular surface albedo and vegetation index mappings (e.g., Rockwood and Cox, 1978; Tarpley et al., 1984; Tucker et al., 1984), and radiation budget studies (e.g., Stephens et al., 1981; Gautier, 1982, 1986). For these applications, the need for accurate calibration of the involved satellite data is easily perceived, and can hardly be overemphasized. To illustrate this, Fig. 1 shows the impact of calibration errors on selected geophysical quantities currently derived from satellite data, namely the normalized vegetation index, the surface albedo and the downward solar irradiance at the surface. The impact is particularly dramatic on the normalized vegetation index (Fig. 1a). For a green biomass level of $660 \mathrm{gm}^{-2}$, a $20 \%$ error in the calibration of the AVHRR visible (channel 1, 0.58-0.68 $\mu \mathrm{m}$ ) and near-infrared (channel 2, 0.73-1.10 $\mu \mathrm{m}$ ) bands translates into a typical $61 \%$ error in the normalized vegetation index. The error rapidly increases with decreasing biomass level, and reaches $114 \%$ for a $260 \mathrm{gm}^{-2}$ biomass level. In the case of the surface albedo (Fig. 1b), a $20 \%$ error in the calibration of the VISSR/VAS visible band $(0.52-0.73 \mu \mathrm{m})$ introduces a similar error in the surface albedo, typically ranging from 19 to $34 \%$ between the extreme cases of white gypsum sand and clear sea water. Finally, it is clear from Fig. 1c that for highly reflecting clouds (i.e., albedo >0.5) even small calibration errors in the VISSR/VAS visible band yield important solar irradiance errors. When monitoring such quantities over long-term periods, for instance in climate studies, the smallest fluctuations are often determinant and must, therefore, be resolved. If the satellite data used in the calculations are not accurately calibrated, not only will small changes not be detected, but the results may also turn out unreliable and misleading (e.g., comparisons of different data sets become nonsensical). 

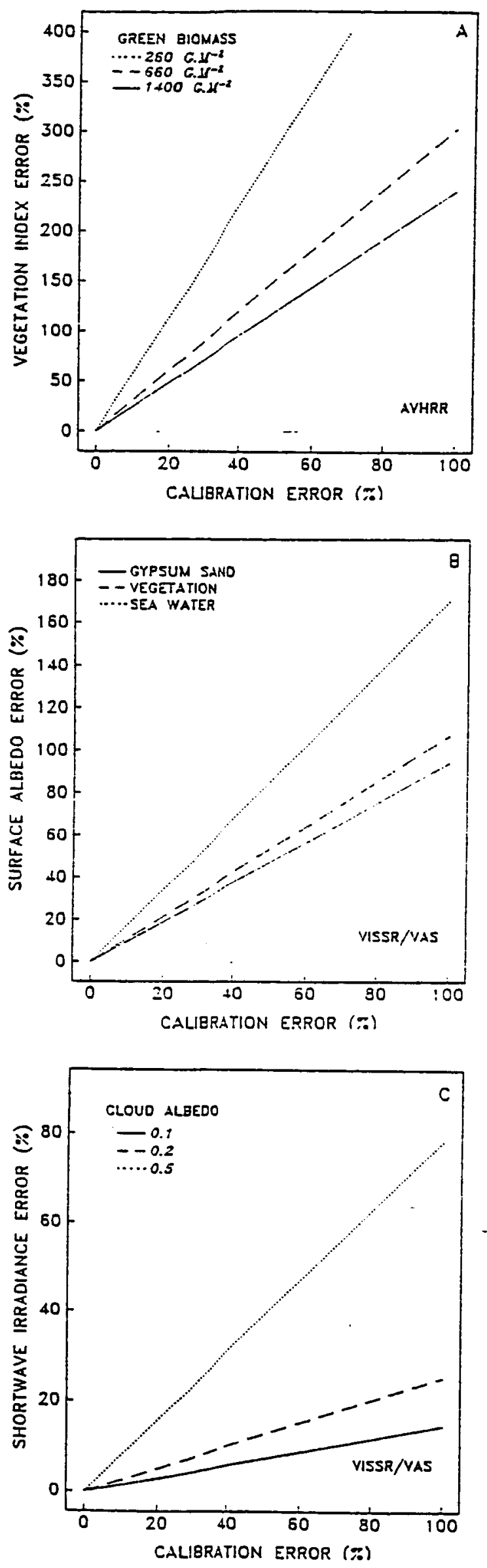

Fig. 1. Sensitivity of vegetation index, surface insolation and ground albedo to calibration errors. 
Careful preflight laboratory calibrations of the VISSR/VAS and AVHRR visible channels were performed, respectively, by the Santa Barbara Research Center (Staff Members, 1980 ) and by ITT Aerospace (Lauritson et al., 1979; Koczor, personal communication). Transfer functions were established to calculate satellite level irradiances from the telemetered digital sensor signals. These functions, however, may not be valid for the data acquired in operations because the orbital satellite environment differs from the steady state laboratory environment and inflight changes occur, as observed with other radiometers. A serious problem is the possibility of dark current from the detectors and zero offsets in the signal conditioning circuitry. Degradation of the optics with time is also not uncommon. It is therefore important to maintain a check-of-calibration for the visible channels while the instruments operate in orbit.

Unfortunately, the AVHRR contains no calibration capabilities other than space viewing for channels 1 and 2. Onboard facilities exist for the VISSR/VAS visible channel (i.e., an optical subassembly with reduced size aperture for directly viewing the sun), but they are not being utilized because of uncertainties in the characteristics of the optical elements.

Several substitutive methods of calibration can yet be envisioned. One can utilize independent and coincident measurements from a suitably equipped high-flying aircraft (e.g., Smith and VonderHaar, 1980; Kriebel, 1981; Hovis et al., 1985) or by a calibrated satellite-borne instrument with similar spectral characteristics (e.g., Smith and Loranger, 1977; Brooks et al., 1984). Another possibility, perhaps the simplest way, as pointed out by Coulson and Jacobowitz (1972), is to use areas on the earth's surface as calibration targets (e.g., Koepke, 1982). This last method is adopted in the present study and is applied to NOAA-7 AVHRR, GOES-5 and GOES-6 VISSR/VAS shortwavelength channels. In this paper, we report on the results obtained for the period from October 1983 to January 1985. 


\section{Method of Calibration}

The first step is to select a suitable calibration target on the earth's surface. Once the target is selected, the intensity of the radiation that is directed to space from the target to the satellite is calculated from the optically acting parameters of the target and of the intervening atmosphere, and is related to the sensor digital output. The procedure is then repeated periodically to monitor eventual time changes in the behavior of the instruments.

The GOES-5 and GOES-6 VISSR/VAS visible channels are actually comprised of eight independent detectors, similar in spectral response (Figs. $2 \mathrm{a}$ and $2 \mathrm{~b}$ ) and sharing the same telescope and scan assembly. The analog signal from the detectors is digitized into 6-bit counts before its transmission to the ground, but two bits are added to each count at the NOAA Command and Data Administration (CDA) station in Wallops Island (i.e., users have access to 8-bit coded data with, however, only 6-bit accuracy). While the detectors respond linearly to incident irradiance, the digitizing scale is not linear, but purposely adjusted to keep the signal-to-noise ratio linear with respect to digital count, i.e., the digital count is proportional to the square root of the voit response (Fig. 3). Thus, the irradiance sensed by each VISSR/VAS visible detector varies as

$$
E=G X^{2}+I
$$

where $E$ is the irradiance of the target at count value $X$, and $G$ and $I$ are calibration constants. It is important to emphasize that this relationship, currently adopted by investigators, is only approximate. At count value 48 on the 6-bit scale, for instance, a $7 \%$ error in $E$ is introduced when using the best fit of Fig. 3 for the analog-digital transfer function.

As opposed to GOES-5 and GOES-6 VISSR/VAS visible channels, for which the spectral response of the detectors defines the optical bandwidth, the wavelength range of NOAA-7 AVHRR channels 1 and 2 (Fig. 2c) is controlled by a filter. The voltage output of the detectors (one per channel), converted into 10-bit counts aboard the spacecraft, is linear as a function of sensed irradiance (Lauritson et al., 1979), i.e.,

$$
E=G X+I
$$

describes the relationship between counts and irradiances.

To determine the "constants" $G$ and $I$, two calibration points (two $X, E$ pairs) are needed. The White Sands Monument area in New Mexico (hereafter referred to as White 


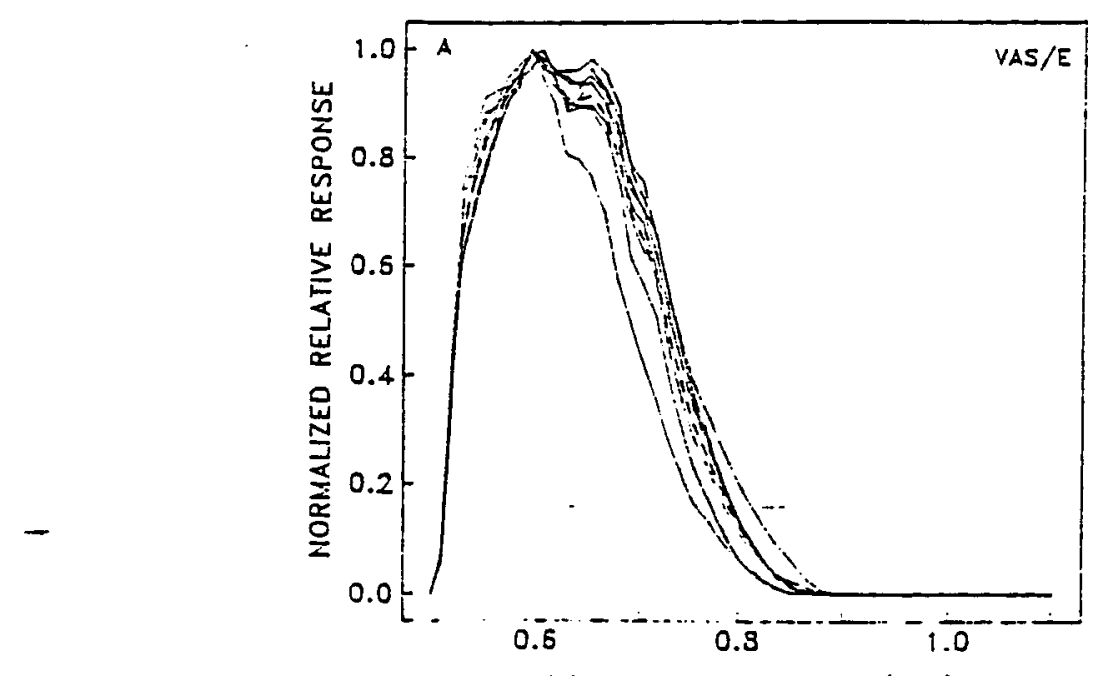

ORIEINAL PAGE IS
Or POOR QUALITY

SPECTRAL WAVELENGTH ( $\mathrm{um})$
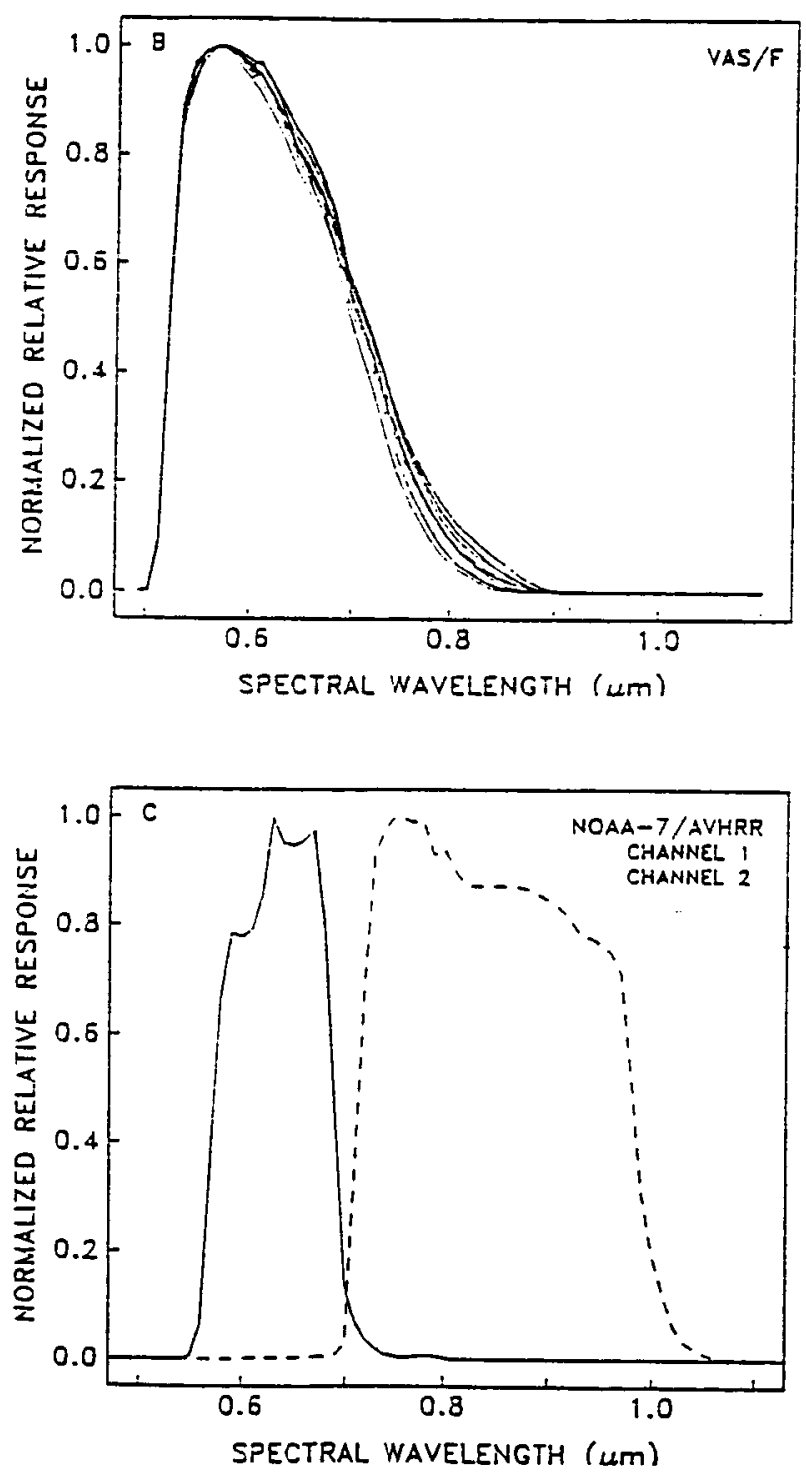

Fig. 2. Spectral response of the solar channels. 


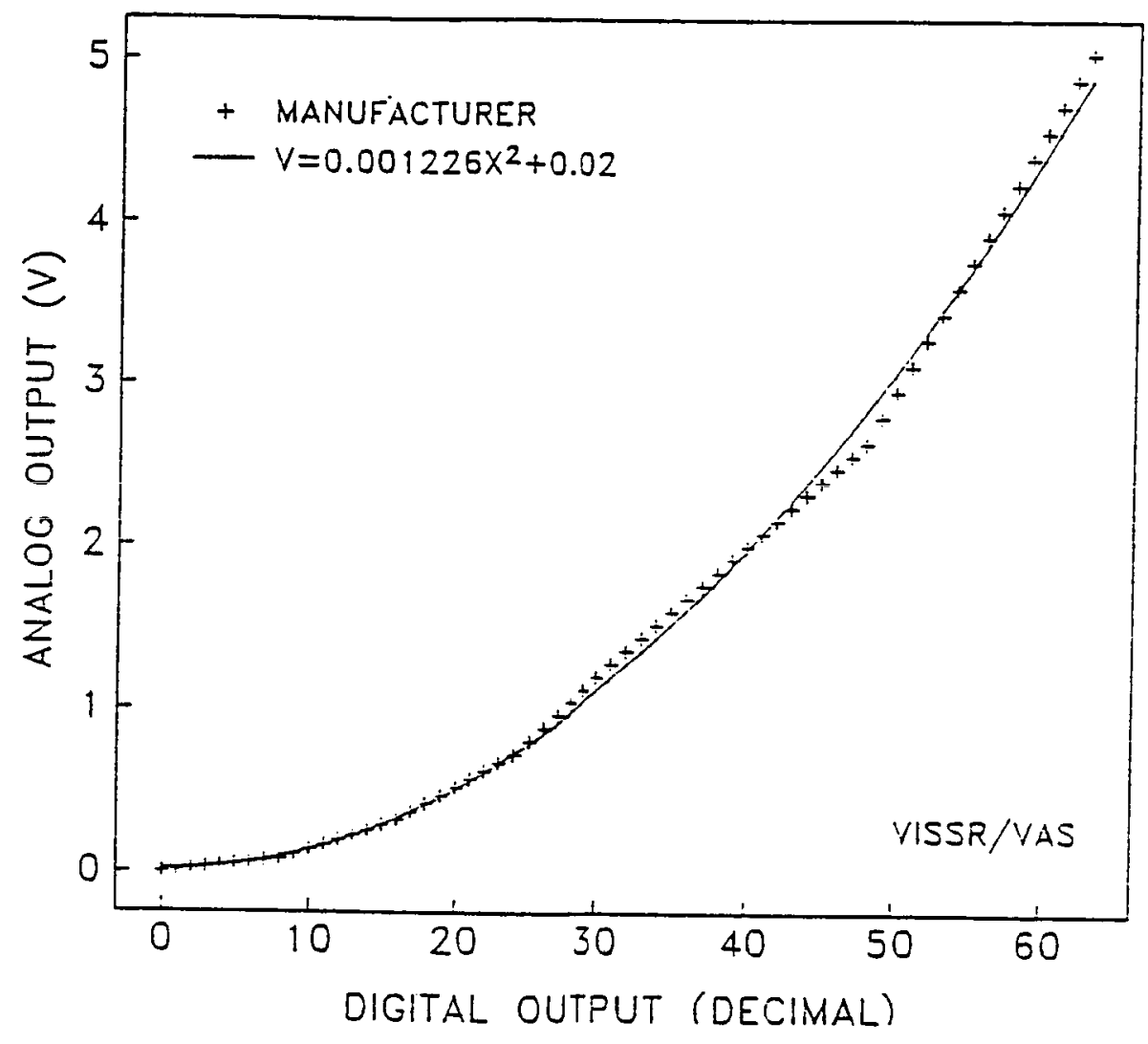

Fig. 3. Analog-digital VISSR/VAS transfer function. 
Sands), located at $32^{\circ} 52^{\prime} \mathrm{N}, 106^{\circ} 17^{\prime} \mathrm{W}$ and $1.22 \mathrm{~km}$ altitude, which is highly reflective, provides a suitable high calibration point. First, the area is large, approximately $30 \mathrm{~km}$ in diameter, providing multiple sampling points and minimizing edge and environmental effects. Second, its reflectance properties are well-known, stable in time and fairly well approximate those of a Lambert surface, reducing bidirectional effects and simplifying the radiative transfer modeling. Finally, favorable atmospheric conditions mostly prevail in that region (minimum cloudiness, relatively low aerosol content). The area, however, is not uniform horizontally. A high water table keeps the very low parts in a permanently wet state, modifying its reflectance. Part of the area is covered by dunes, and their shading is difficult to compensate for, thereby restricting the observations to conditions of small solar and viewing zenith angles. The selection of small zenith angles is also important, as we shall see, to reduce uncertainties resulting from unknown atmospheric characteristics. The second calibration point, at practically zero intensity value, is provided by space.

As evidenced in Figs. $2 a$ and $2 b$, the spectral response of GOES-5 and GOES-6 VISSR/VAS visible detectors may differ in the longwavelength half power point by as much as $0.1 \mu \mathrm{m}$, which suggests that the calibration be effected separately for each detector. However, an average calibration of the eight detectors is accomplished for each satellite, using average count data and average spectral responses (also shown in Figs. 2a and $2 \mathrm{~b}$ ). This procedure is justified because the data which are made available to users by NOAA CDA have been processed to eliminate displeasing stripes that arise from differences among the detectors. The output of the eight detectors is normalized to one of the detectors, and the normalization is readjusted every couple of weeks. Moreover, in some cases, different detectors are used as the normalizing detector. In other words, the data calibrated one week may be out of calibration the following week. Consequently, our vicarious calibration of the GOES-5 and GOES-6 VISSR/VAS visible channels will be performed every two weeks and, for convenience, we shall proceed in the same way for NOAA-7 AVHRR channels 1 and 2.

\section{Radiative Transfer Model}

\section{a. Model}

In the spectral region of interest, $0.5-1.1 \mu \mathrm{m}$, the sensed energy originates almost entirely from solar radiation which propagates through the atmosphere, is reflected by the surface target and is finally transmitted to the space-borne sensor. The thermal radiation 
emitted by the atmosphere itself is negligible in comparison with the solar component, so it will be ignored.

Solar radiation in the $0.5-1.1 \mu \mathrm{m}$ wavelength range is scattered by air molecules and aerosols, and is absorbed primarily by ozone, water vapor, oxygen and aerosols. Scattering and absorption processes complicatedly interact, but fortunately, gaseous absorption can be treated separately (Deschamps et al., 1983; Tanré et al., 1985). Qualitatively, the arguments are the following. First, ozone is located at altitudes where molecules and aerosols are already very rarified, i.e., the ozone layer is traversed almost without scattering. Ozone, therefore, simply acts at reducing the radiation signal by the direct transmittance (i.e., along a direct path) corresponding to the double passage across the absorbing layer. Second, water vapor absorption occurs at wavelengths greater than 0.85 $\mu \mathrm{m}$, where molecular scattering is insignificant and where only aerosol scattering subsists. Since (1) aerosol scattering is mainly primary and secondary at those wavelengths, and (2) the aerosol phase function presents a pronounced forward peak, the photons practically follow a direct path through the atmosphere. To account for water vapor absorption, it is therefore sufficient to affect the signal that would be observed if the atmosphere were not absorbing by the transmittance along the direct path from the sun to the target and from the target to the satellite. Part of the solar radiation incident at the top of the atmosphere is in fact scattered back into space before any surface reflection takes place. The separation of scattering and absorption processes is more difficult to justify in this case, but remains a reasonable approximation since absorption by atmospheric gases is generally weak or very localized in the $0.5-1.1 \mu \mathrm{m}$ range. This also warrants the same treatment for oxygen absorption, which occurs in a very narrow band near $0.76 \mu \mathrm{m}$, although molecular scattering cannot be considered unimportant at this wavelength.

Thus, the irradiance measured by the space-borne sensor viewing the surface target can be written with a good degree of approximation as

$$
E\left(M, \theta_{0}, \theta, \phi\right) \simeq \int_{0}^{\infty} F_{\lambda} E_{0 \lambda} \cos \theta_{0} t_{g \lambda}\left(\theta_{0}, \theta\right) \rho_{\lambda}^{*}\left(M, \theta_{0}, \theta, \phi\right) d \lambda
$$

where $\lambda$ is wavelength, $\theta_{0}$ and $\theta$ are respectively the solar and viewing zenith angles, $\phi$ is the relative azimuth angle, $F_{\lambda}$ is the spectral response of the detectors or filters, $E_{0 \lambda}$ is the extraterrestrial solar irradiance (in $\mathrm{Wm}^{-2} \mu \mathrm{m}^{-1}$ ), $t_{g \lambda}$ is the transmittance due to absorbing gases, and $\rho_{\lambda}^{*}$ is an equivalent reflectance of the target $M$. 
The radiation geometry is depicted in Fig. 4. The equivalent reflectance $\rho_{\lambda}^{*}$ is defined as

$$
\rho_{\lambda}^{*}\left(M, \theta_{0}, \theta, \phi\right)=\frac{E_{\lambda}^{\prime}\left(M, \theta_{0}, \theta, \phi\right)}{E_{0 \lambda} \cos \theta}
$$

where $E_{\lambda}^{\prime}$ is the irradiance that would be sensed if the air molecules were non-absorbing. In other words, $\rho_{\lambda}^{*}$ takes into account aerosol and molecular scattering and aerosol absorption, but excludes gaseous absorption. The study deals with monochromatic quantities, but subscript $\lambda$ will be omitted afterward to simplify the notation.

The equivalent reflectance $\rho^{*}$ is modeled according to Tanré et al., (1979):

$$
\begin{aligned}
\rho^{*}\left(\theta_{0}, \theta, \phi\right) \simeq \rho_{a}\left(M, \theta_{0}, \theta, \phi\right)+\frac{1}{1-(\rho)\left(M, \theta_{0}, \theta, \phi\right) r} \\
{\left[A \rho\left(M, \theta_{0}, \theta, \phi\right)+B \bar{\rho}\left(M, \theta_{0}, \theta, \phi\right)+C\langle\rho\rangle\left(M, \theta_{0}, \theta, \phi\right)\right] }
\end{aligned}
$$

where $\rho_{a}$ is the intrinsic atmospheric reflectance (i.e., the contribution of the signal backscattered into space without surface reflection), $r$ is the spherical albedo of the atmosphere, $\rho$ is the bidirectional reflectance of the target, and $\bar{\rho}$ and $\langle\rho\rangle$ are average angular and environmental reflectances. In Eq. $5, A, B$ and $C$ are development coefficients characterizing the relative importance (or weight) of reflectances $\rho, \bar{\rho}$ and $\langle\rho\rangle$. Detailed expressions for $A, B$ and $C$ can be found in Tanré et al., (1979).

The reflectance $\bar{\rho}$ corresponds to photons going diffusely from the sun to the surface, and then from the surface directly into space, i.e.,

$$
\bar{\rho}\left(M, \theta_{0}, \theta, \phi\right)=\frac{\int_{0}^{2 \pi} \int_{0}^{-\pi / 2} \rho\left(M, \theta^{\prime}, \theta, \phi^{\prime}-\phi\right) E_{d}^{-}\left(\theta_{0}, \theta^{\prime}, \phi^{\prime}\right) \cos \theta^{\prime} \sin \theta^{\prime} d \theta^{\prime} d \phi^{\prime}}{\int_{0}^{2 \pi} \int_{0}^{-\pi / 2} E_{d}^{-}\left(\theta_{0}, \theta^{\prime}, \phi^{\prime}\right) \cos \theta^{\prime} \sin \theta^{\prime} d \theta^{\prime} d \phi^{\prime}}
$$

where $E_{d}^{-}$is the downward diffuse irradiance at the surface for $\rho=0$.

The reflectance $\langle\rho\rangle$ represents photons that have sustained reflections from the area adjacent to the target, and appears in Eq. 5 because the reflectance of this surrounding background a priori differs from that of the target. It is defined as

$$
\langle\rho\rangle\left(M, \theta_{0}, \theta, \phi\right)=\frac{\int_{-\infty}^{+\infty} \int_{-\infty}^{+\infty} \cos \theta_{0} E_{0} \rho\left(M^{\prime}, \theta^{\prime}, \theta, \phi\right) t\left(\theta, x^{\prime}, y^{\prime}\right) d x^{\prime} d y^{\prime}}{\int_{0}^{2 \pi} \int_{0}^{-\pi / 2} E_{d}^{-}\left(\theta_{0}, \theta^{\prime}, \phi^{\prime}\right) \cos \theta^{\prime} \sin \theta^{\prime} d \theta^{\prime} d \phi^{\prime}}
$$

where $t\left(\theta, x^{\prime}, y^{\prime}\right)$ denotes the efficiency of a point $M^{\prime}\left(x^{\prime}, y^{\prime}\right)$ with horizontal coordinates $x^{\prime}$ and $y^{\prime}$. 


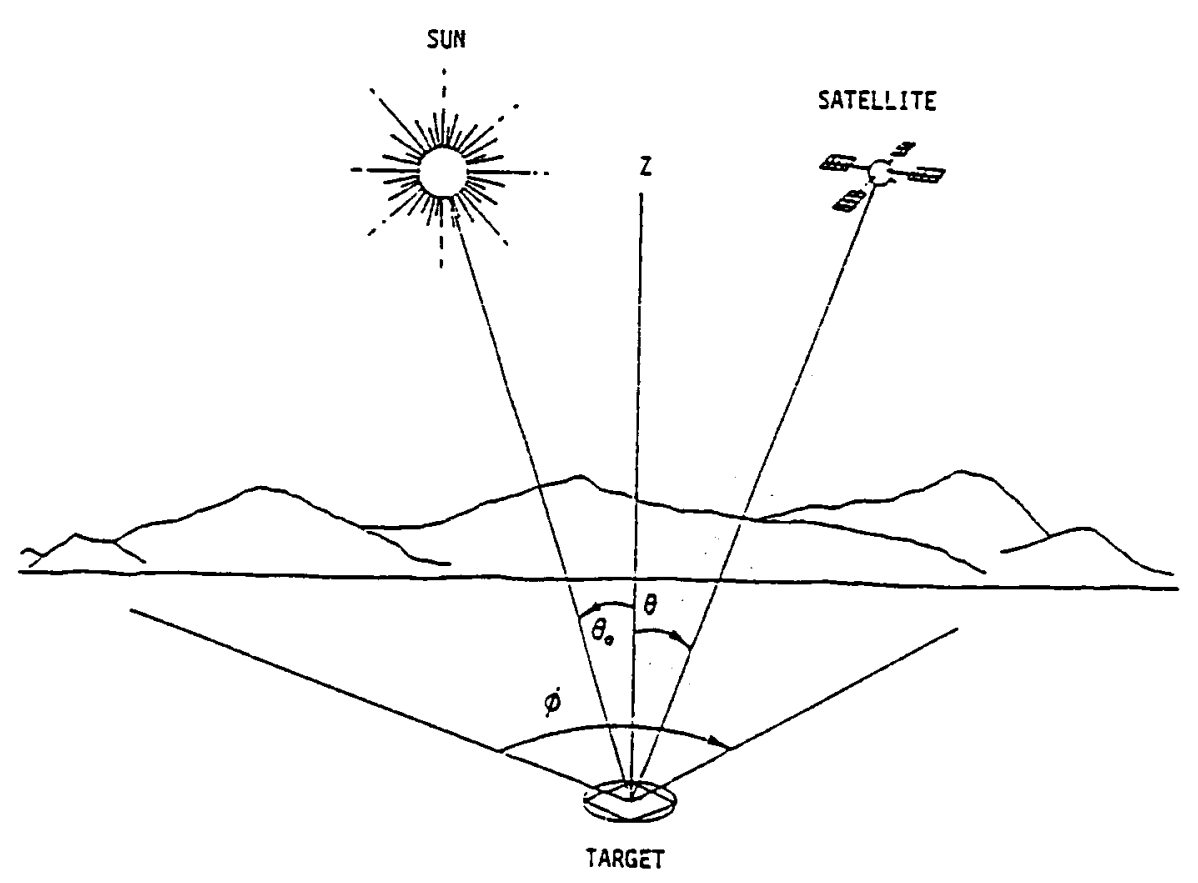

Fig. 4. Radiation geometry. 


\section{b. Solar irradiance}

The solar irradiance incident at the top of the atmosphere is taken from Neckel and Labs (1984) and corrected for earth-sun distance variations during the course of the year. The total irradiance in the different channels, as obtained by convolving Neckel and Labs' (1984) data with the respective response functions, is given in Table 1. Clearly, more energy flux is available in the spectral domain of the VISSR/VAS channels, for the reason that their peak of transmission is closer to the wavelength of maximum solar emission, and also because their bandwidth is relatively large. For AVHRR channel 2, the large bandwidth of the filter partly compensates for the smaller energy flux emitted by the sun in the near-infrared. In order to facilitate the conversion of total irradiances into monochromatic irradiances, we also listed in Table 1 the values of the integral over wavelength of the different filter functions.

\section{c. Atmospheric functions}

To evaluate the gaseous transmittance $t_{g}$, water vapor, oxygen and ozone are considered separately. Following Tanré et al., (1985), the individual transmittances are parameterized as a function of air mass and vertically integrated amount of absorber. The statistical band models of Goody (1964) for water vapor and Malkmus (1983) for ozone and oxygen are used in the calculations. The spectral resolution is $20 \mathrm{~cm}^{-1}$, which allows a precise description of the phenomena (i.e., absorption strength and overlapping of bands). To account for the dependence of the absorption coefficients on temperature and pressure, the CurtisGodson approximation is employed with the US62 standard atmosphere of McClatchey (1971). When absorption by several gases takes place, $t_{g}$ is computed as the product of each separate transmittance.

The atmospheric functions $\rho_{a}, r$ and the coefficients $A, B$ and $C$ (which also depend on the characteristics of the atmosphere) were calculated by Tanré et al., (1985) for different experimental conditions and various atmospheric models. Fairly accurate analytical formulas were established, which give $\rho_{a}, r, A, B$ and $C$ as a function of viewing geometry and optical thickness of the atmosphere (or turbidity). As an alternative, the surface visibility, a parameter routinely measured at all weather stations, may be used instead of the turbidity. This approach is appealing because turbidity is only measured at a few sites, and is selected for the present study. One should note, however, that the correspondance between visibility and turbidity is only a rough inverse proportionality (thick layers 
Table 1. Extraterrestrial solar irradiance in the different channels and integrated filter functions.

\begin{tabular}{ccc}
\hline Satellite/Sensor & $\begin{array}{c}\int_{0}^{\infty} F_{\lambda} d \lambda \\
(\mu m)\end{array}$ & $\begin{array}{c}\int_{0}^{\infty} F_{\lambda} E_{0 \lambda} d \lambda \\
\left(W m^{-2}\right)\end{array}$ \\
\hline GOES-5 VISSR/VAS & 0.199 & 323.31 \\
GOES-6 VISSR/VAS & 0.187 & 307.88 \\
NOAA-7 AVHRR/1 & 0.107 & 175.96 \\
NOAA-7 AVHRR/2 & 0.243 & 255.82 \\
\hline
\end{tabular}


of aerosols may exist aloft with a very clear atmosphere at the surface). Because of the lack of precise knowledge of the aerosol scattering properties at White Sands (i.e., sizefrequency distribution, refractive index), we opted, among diverse aerosol models available, for the continental model of the International Radiation Commission (WCP, 1983). Although this model may not represent what actually occurs at White Sands, it certainly remains useful in setting limits to what might be reasonably expected.

\section{d. Surface reflectance}

The bidirectional reflectance of White Sands $\rho$ was measured in laboratory by Walraven and Coulson (1972) for dry and wet conditions. In-situ measurements were also carried out by Hovis (1966), but they are not applicable to the present study. Hovis (1966) employed a spectrophotometer that measured monochromatic energy in a direction normal to the surface and, consequently, his results do not represent the range of angular conditions encountered in the real world (the White Sands reflectance is a relatively strong function of the angle of incidence). In fact, the energy collected by the integrating sphere of the instrument is reflected in all directions into the hemisphere in front of the sample surface, i.e., angular effects are lost.

Fig. 4 summarizes the main features reported by Walraven and Coulson (1972). For dry sand, the spectral reflectance curve (Fig. 5a) is characterized by a rapid increase to $0.6 \mu \mathrm{m}$, followed by a slight dip around $0.7 \mu \mathrm{m}$, continued by a barely perceptible increase beyond $0.8 \mu \mathrm{m}$. For wet sand, the entire reflectance curve is displaced toward lower values by about 0.1 , or $15 \%$. The slope is steeper below $0.6 \mu \mathrm{m}$, and the depression at 0.7 $\mu \mathrm{m}$ is more pronounced. Above $0.8 \mu \mathrm{m}$, as opposed to dry sand, the curve exhibits a negative declivity caused by water vapor absorption in the $0.94 \mu \mathrm{m}$ band. The shape of the curves below $0.6 \mu \mathrm{m}$ is consistent with the general finding that, for sandy textured soils, the reflectance sharply drops in magnitude at wavelengths smaller than the average particle size. In interpreting Fig. 4a, however, one should bear in mind that the portion of the curves extending beyond $0.9 \mu \mathrm{m}$ results from a linear extrapolation of Walraven and Coulson's (1972) data. This questionable procedure is at least consistent with Hovis' (1966) measurements, which exhibit a fairly linear comportment in the 0.8-1.0 $\mu \mathrm{m}$ domain.

Important angular effects at $0.7 \mu \mathrm{m}$ are clearly seen in Figs. $4 \mathrm{~b}$ and $4 \mathrm{c}$. Variations with the solar zenith angle (or equivalently with the viewing zenith angle because of the reciprocity principle) are particularly significant for dry sand (Fig. 5b), i.e., $15 \%$ when 

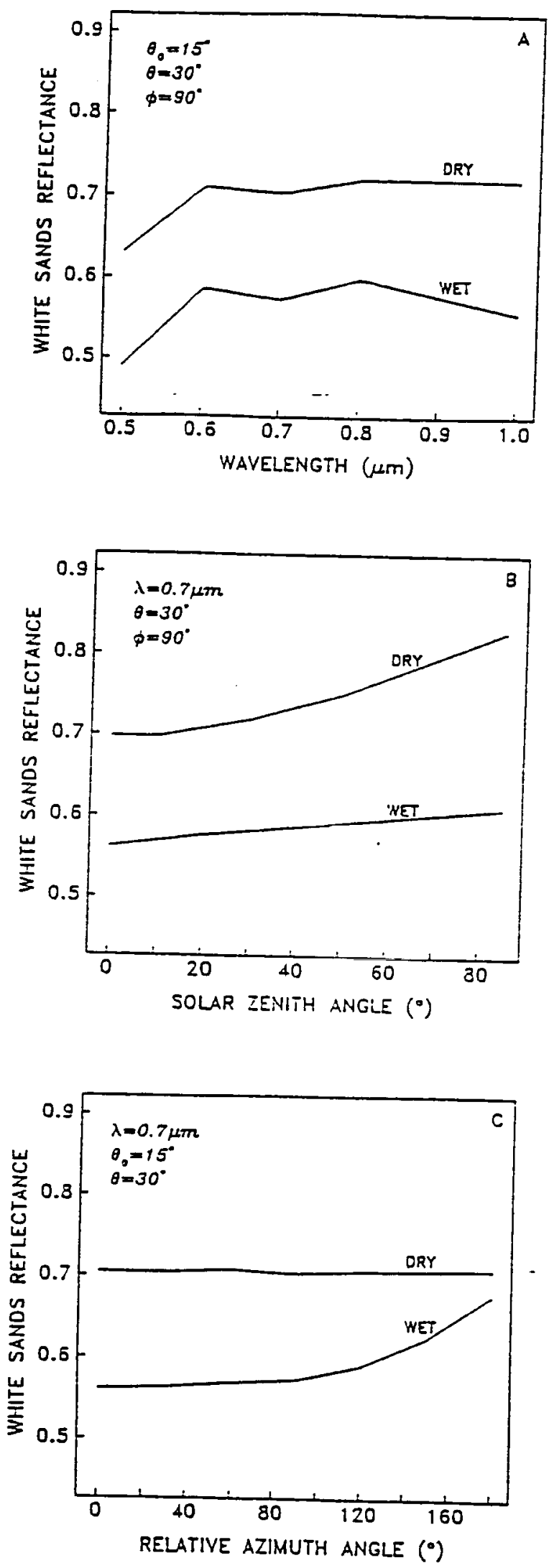

Fig. 5. Reflectance of White Sands as a function of wavelength, solar and viewing zenith angles, and relative azimuth angle (after Walraven and Coulson, 1972). 
passing from 0 to $60^{\circ}$ for the specified conditions. The reflectance curve for wet sand is characterized by a nearly constant slope, and the overall effect is more moderate (only a $5 \%$ change in the $0-60^{\circ}$ range). The behavior is similar at other wavelengths (not shown here): generally, an isotropic distribution is observed at small zenith angles, all the more so as the wavelength decreases. Almost no dependence on the relative azimuth angle is discerned for dry sand (Fig. 5c). This is in agreement with the previous statement, because the zenith angles considered in Fig. 4c are small. At larger zenith angles, a specular component superimposes the diffuse contribution, its importance increasing as the principal plane is approached (i.e., $\phi=180^{\circ}$ ). Even for solar and viewing zenith angles of $15^{\circ}$ and $30^{\circ}$, however, the wet sand data (Fig. 5c) shows definite evidence of a specular component.

Inspection of Fig. 5 clearly reveals the strong influence of surface moisture upon White Sands reflectance, which therefore ought to be carefully taken into account. Unfortunately, soil moisture is not currently measured at White Sands and, anyhow, the usefulness of punctual or isolated soil moisture data, even recorded when the satellite views the target, is debatable. A correction is therefore estimated with the help of gravimetric measurements performed by Williamson (1975) during a one-year period. These measurements are reproduced in Fig. 6 for two sites, one located in the flat portion of the area and the other in the white gypsum sand dunes. Clearly distinguishable in Fig. 5 is a seasonal dependence, with minimum values during summer (about $10 \%$ ) and maximum values during winter (typically $30 \%$ in the flats and $20 \%$ in the dunes). Short-term variations are also evident, and can be of importance (e.g., $20 \%$ over a few day period around Julian day 200 in the flats). The yearly averages are $21.4 \pm 5.3(1 \sigma) \%$ in the flats and $17.8 \pm 3.2(1 \sigma) \%$ in the dunes. Since the standard deviations for the entire one-year period translate into surface reflectance changes of less than $0.6 \%$, we concluded that the yearly averages would adequately represent the actual moisture conditions at White Sands. We are aware, however, that this procedure is somewhat arbitrary, because of the high spatial and temporal variability of surface moisture. Also, the measurements of Williamson (1975), even though they correspond to clear or partly cloudy skies without rain, were taken only near noon (local), and we do not know about the atmospheric events preceding these measurements: A better way to proceed would certainly consist of directly measuring the reflectance of White Sands at the very moment of the satellite pass, but such a practice is beyond the scope of this study. Although the distinction between flats and dunes in not always apparent in the satellite data, we decided, for consistency, to restrict our analysis to the 
ORIGINAL PAGE IS

G. POOR QUALTTY

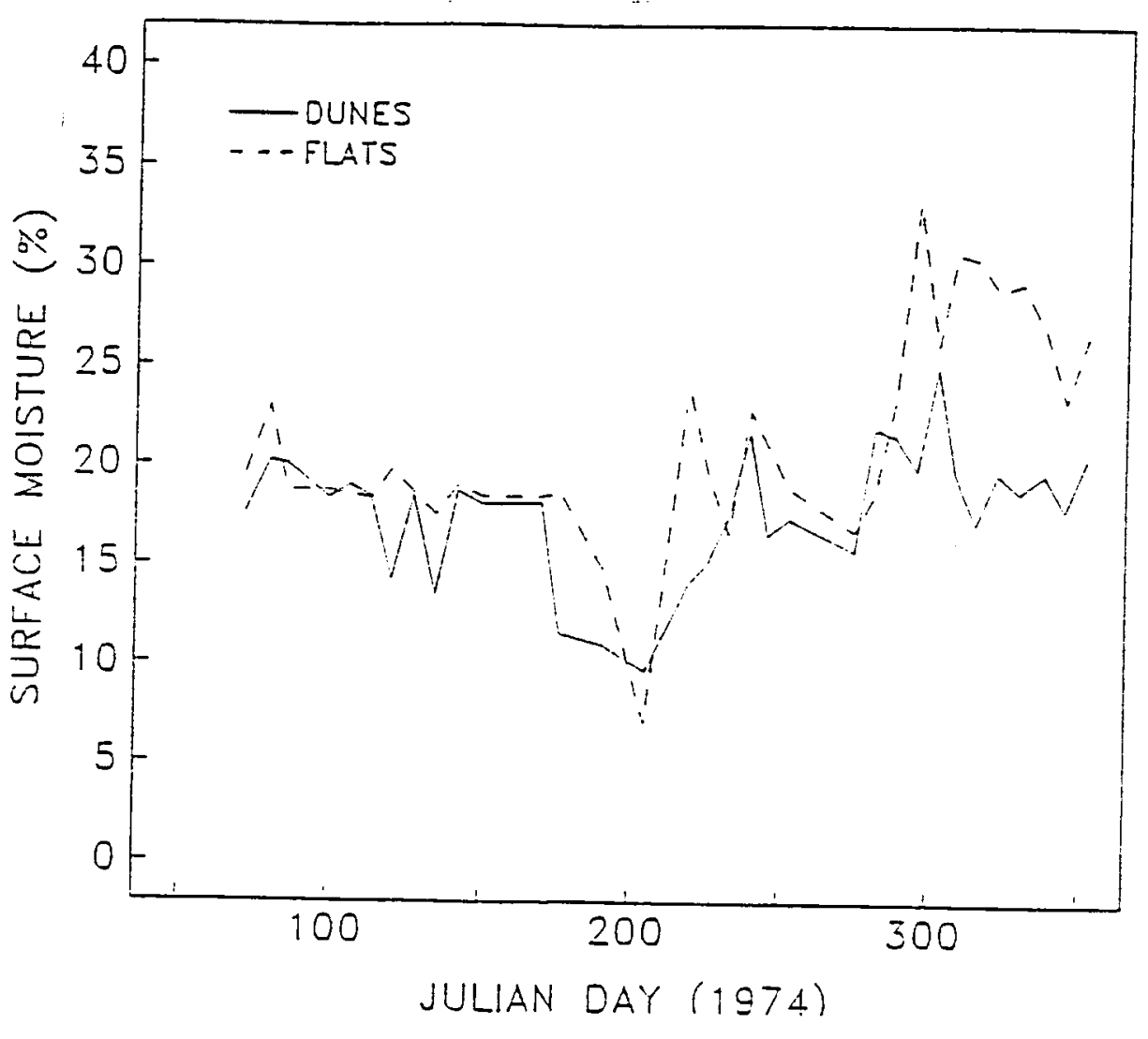

Fig. 6. Evolution of the soil moisture at White Sands during the course of the year (after Williamson, 1975). 
dunes area. In the simulations, consequently, the surface moisture is fixed at the yearly average value of $17.8 \%$. Since Walraven and Coulson's (1974) data indicate that, in some degree, the reflectance of white gypsum sand varies linearly when the sand dries from a saturated state, the moisture effect at the intermediate value of $17.8 \%$ is simply computed by linear interpolation.

\section{e. Average reflectances}

The average reflectances $\bar{\rho}$ and $\langle\rho\rangle$ are laborious to evaluate, because their calculation (via Eqs. 6 and 7) involves angular integration of the diffuse irradiance $E_{d}^{-}$and requires precise knowledge of the spatial variations of the target reflectance. Fortunately, the difficulty can be obviated. In order to do this, let us first examine the relative importance of development coefficients $A, B$ and $C$ (Fig. 7). One notices that, in the wavelength range of interest, $A$ largely surpasses $B$ and $C$ in magnitude for clear atmospheres (i.e., for visibilities greater than $23 \mathrm{~km}$ ) and not-too-horizontal sightings. In other words, the contribution of the signal directly reflected by the ground, which naturally involves the true reflectance of the target $\rho$, is highly preponderant for sufficiently clear atmospheres. Second, the spherical albedo of the atmosphere $r$ does not exceed the value of 0.2 , even for considerable aerosol loadings (Fig. 8). Third, $\bar{\rho}$ and $\langle\rho\rangle$ do not notably depart from $\rho$. As shown in Fig. $9,(\rho-\bar{\rho}) / \bar{\rho}$ lies below $5 \%$ in most situations. It decreases with increasing solar zenith angle and, for the conditions specified in the figure, diminishes to zero at solar zenith angles of $40-50^{\circ}$. Beyond these values, the difference is negative (i.e., $\bar{\rho}<\rho)$. This feature simply reflects the influence of the specular component of the White Sands reflectance, which is important at large solar zenith angles, even in the direction perpendicular to the principal plane. In the computation of $\bar{\rho}, E_{d}^{-}$was expressed as the sum of an isotropic component and a Dirac function (see Tanré et al., 1983), which makes $\bar{\rho}$ directly proportional to $\rho$. For given zenith angles, $(\bar{\rho}-\rho) / \bar{\rho}$ decreases with increasing relative azimuth angles, but moderatly in the case of Fig. 9 because the dependence of $\rho$ on $\phi$ is small at $\theta_{0}=15^{\circ}$ and $\theta=30^{\circ}$ (relatively small zenith angles). The variations with the viewing zenith angle, almost negligible at $\lambda=0.5 \mu \mathrm{m}$ in Fig. 9 , are notable at $\lambda=0.9 \mu \mathrm{m}$, and become more important as $\theta_{0}$ and $\phi$ increase. Because $E_{d}^{-}$appears in both the numerator and denominator of (6), the dependence of $\bar{\rho}$-and a fortiori of $(\bar{\rho}-\rho) / \bar{\rho}$-upon the atmospheric visibility is partly smoothed out. 

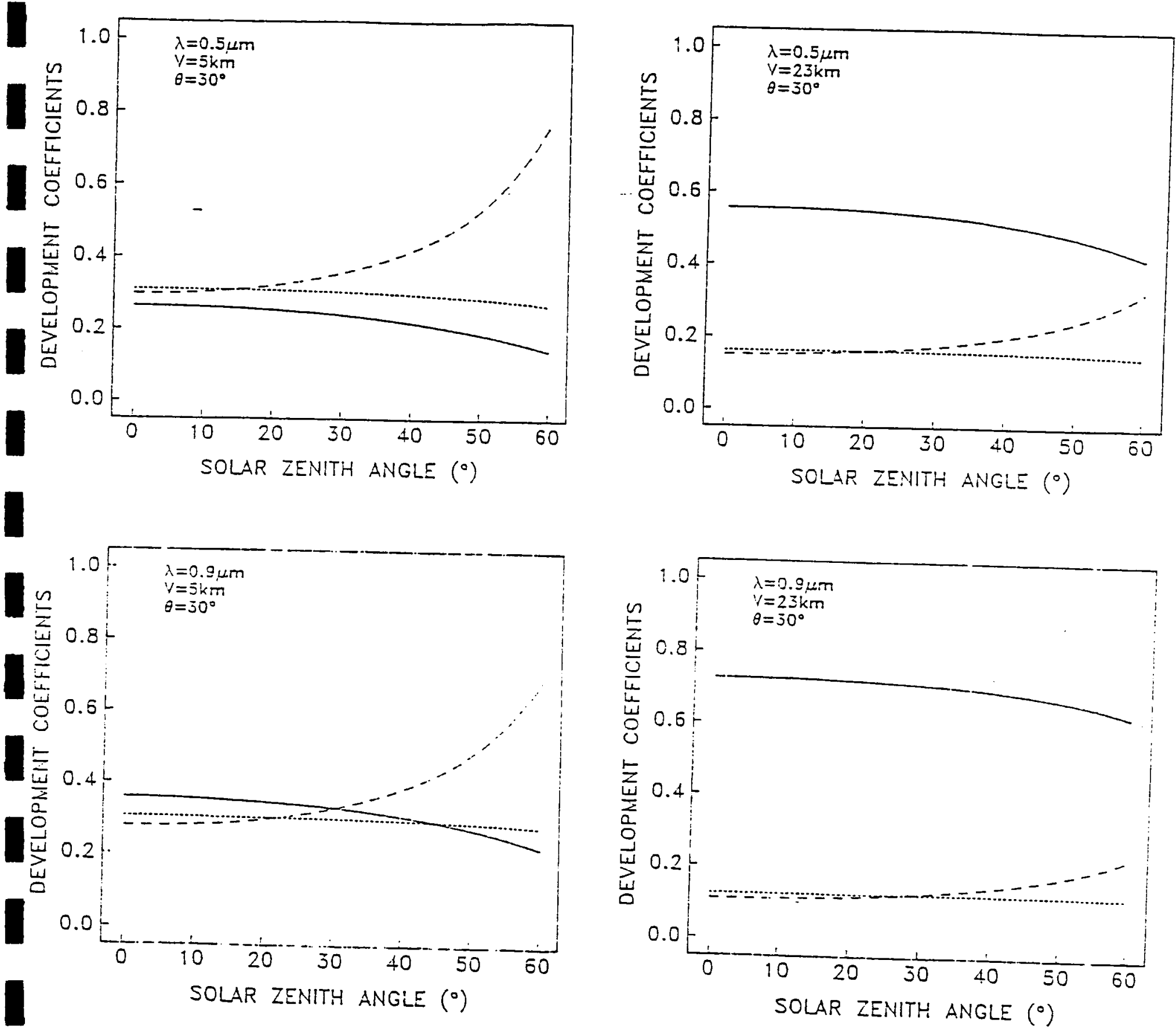

Fig. 7. Development coefficients $A$ (solid lines), $B$ (dashed lines) and $C$ (dotted lines) for different wavelengths, model atmospheres and geometrical conditions. 


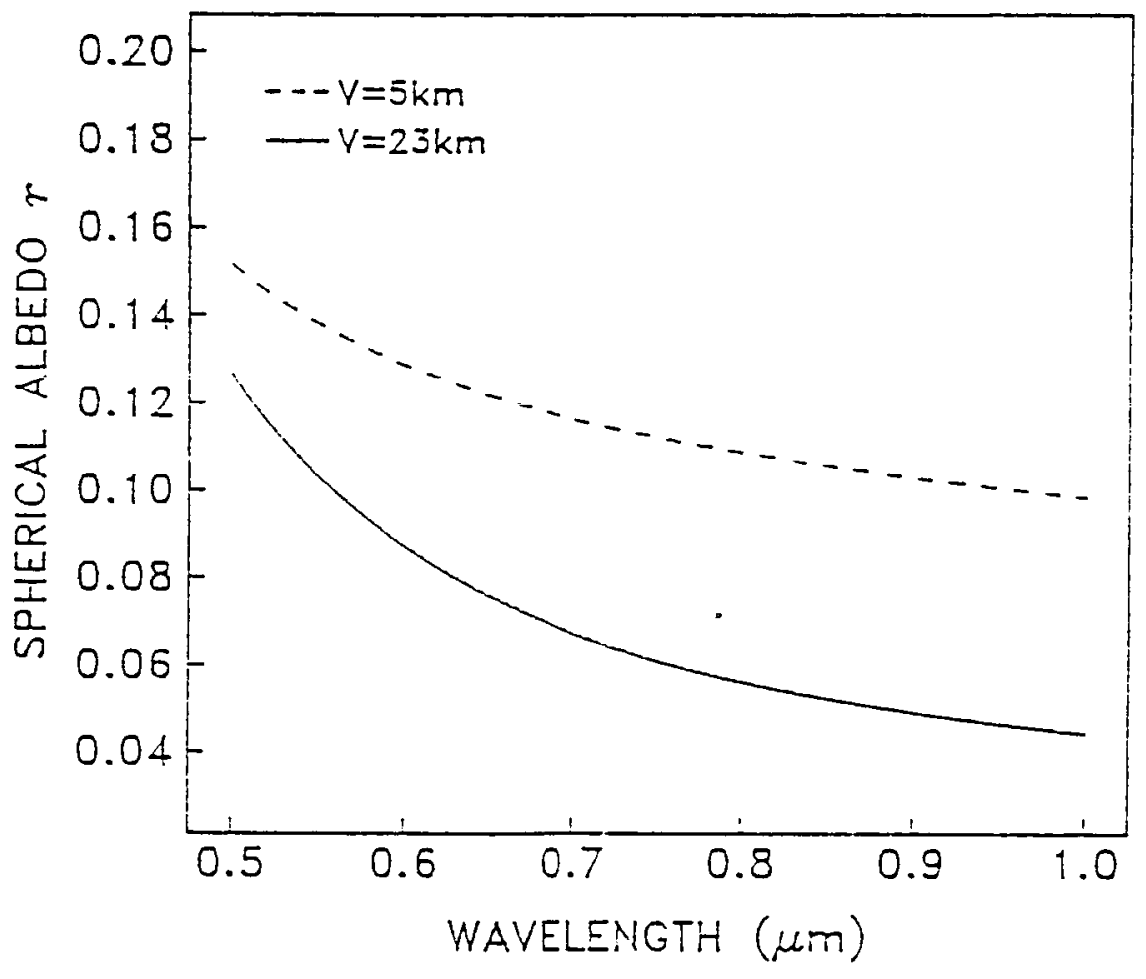

Fig. 8. Spectral dependence of the spherical albedo of the atmosphere. 

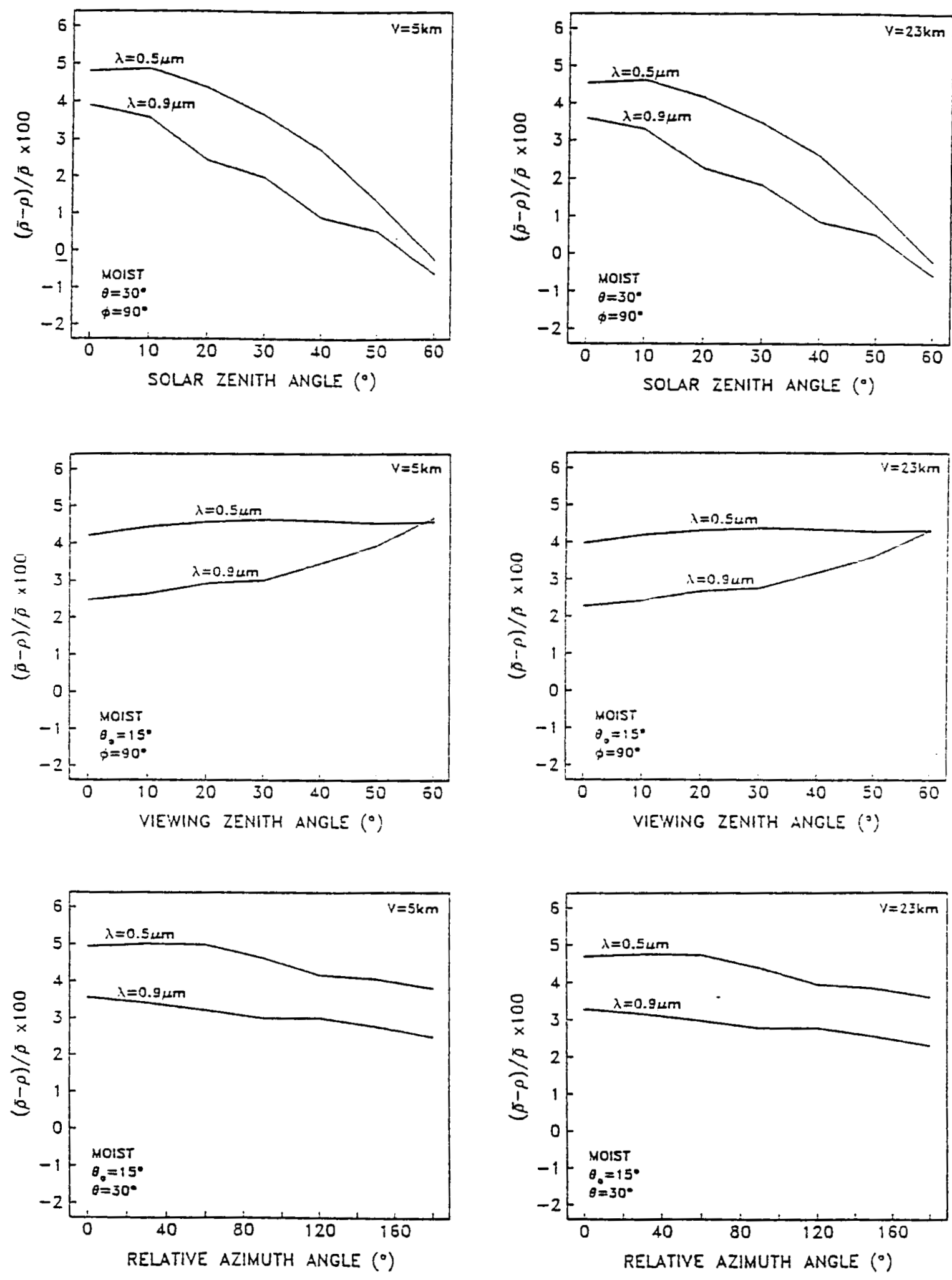

Fig. 9. Departure from angular average reflectance $\bar{\rho}$ of $\rho$ for different wavelengths, model atmospheres and geometrical conditions. 
ORIGINAL. PAGE IS

OF. POOR QuAuTr
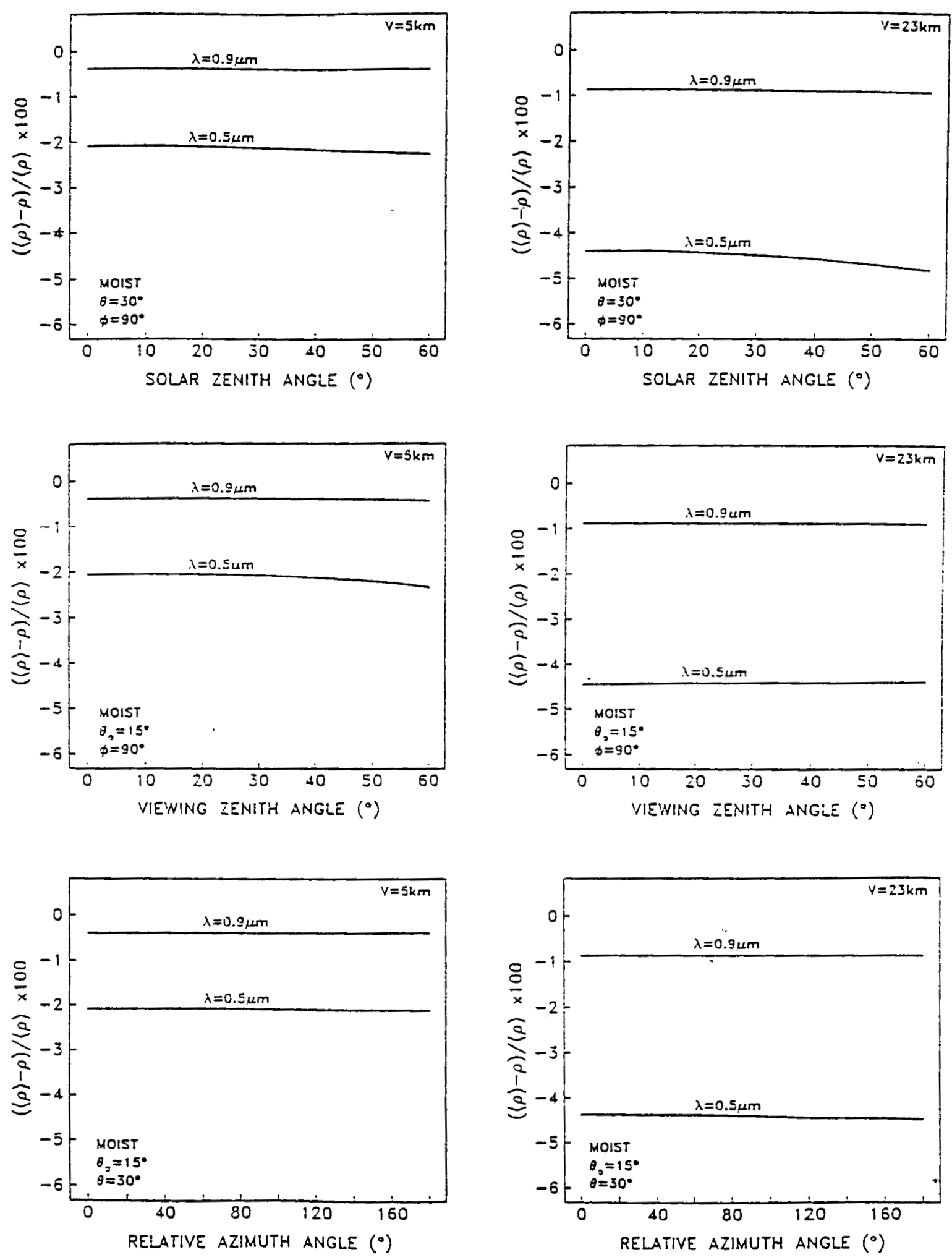

Fig. 10. Same as Fig. 9, but $\langle\rho\rangle$ instead of $\bar{\rho}$. 
The angular variations of $(\langle\rho\rangle-\rho) /\langle\rho\rangle$ (Fig. 10) are almost nonexistent, a direct consequence of the large horizontal extent of the White Sands area. The atmospheric visibility, a parameter of minor importance at $\lambda=0.9 \mu \mathrm{m}$, significantly affects $\langle\rho\rangle$ and, hence, $(\langle\rho\rangle-\rho) /\langle\rho\rangle$ at $\lambda=0.5 \mu \mathrm{m}$, because scattering processes are more operative at smaller wavelengths. Because the reflectance of the White Sands environment is lower than that of White Sands, $\langle\rho\rangle$ is smaller than $\rho$, and the magnitude of the difference increases with the visibility (with fewer aerosols, more photons originating from the environment are scattered in the direction of observation). This is clearly seen in Fig. 10: $(\langle\rho\rangle-\rho) /\langle\rho\rangle$ decreases from about $-0.5 \%$ to $-5 \%$ when the visibility is increased from 5 to $23 \mathrm{~km}$. To evaluate $\langle\rho\rangle$, White Sands was assumed to be a circular target, $30 \mathrm{~km}$ in diameter, surrounded by an homogeneous background of reflectance $\rho_{b}=0.3$ and, following Deschamps et al. (1983), $\langle\rho\rangle$ was written as a linear combination of $\rho$ and $\rho_{b}$.

The preceding considerations, and the fact that $(\bar{\rho}-\rho) / \bar{\rho}$ and $(\langle\rho\rangle-\rho) /\langle\rho\rangle$ are of opposite signs, strongly incline one to replace $\bar{\rho}$ and $\langle\rho\rangle$ in Eq. 5 by $\rho$. In so doing, the modeling of the apparent reflectance $\rho^{*}$ is drastically simplified and reduces to the case of an homogeneous Lambertian surface. The validity of the procedure is substantiated in Fig. 11, which shows the resulting error on $\rho^{*}$. Clearly, the error mainly depends on atmospheric visibility and zenith angles, and does not exceed $\pm 1 \%$ in most conditions. The apparent reflectance $\rho^{*}$ is either underestimated or overestimated, depending on the visibility and on the radiation geometry. This interestingly indicates that, by neglecting the influence of the environment, the selection of clear atmospheres might not be optimum, particularly for those channels whose peak of transmission is located near $0.5 \mu \mathrm{m}$. In the presense of hazy atmospheres, however, the errors introduced by our insufficient knowledge of aerosol optical properties are increased. Fig. 11 also indicates that $\Delta \rho^{*} / \rho^{*}$ is generally reduced at small zenith angles. Such angles are also favored to minimize errors arising from uncertainties in the characteristics of the atmosphere overlying the target (the atmospheric path is smaller). In many cases, however, and this is especially true for a sun-synchronous satellite, it will not be possible to meet the conditions (visibility, radiation geometry) that correspond to the most desirable compromise. 

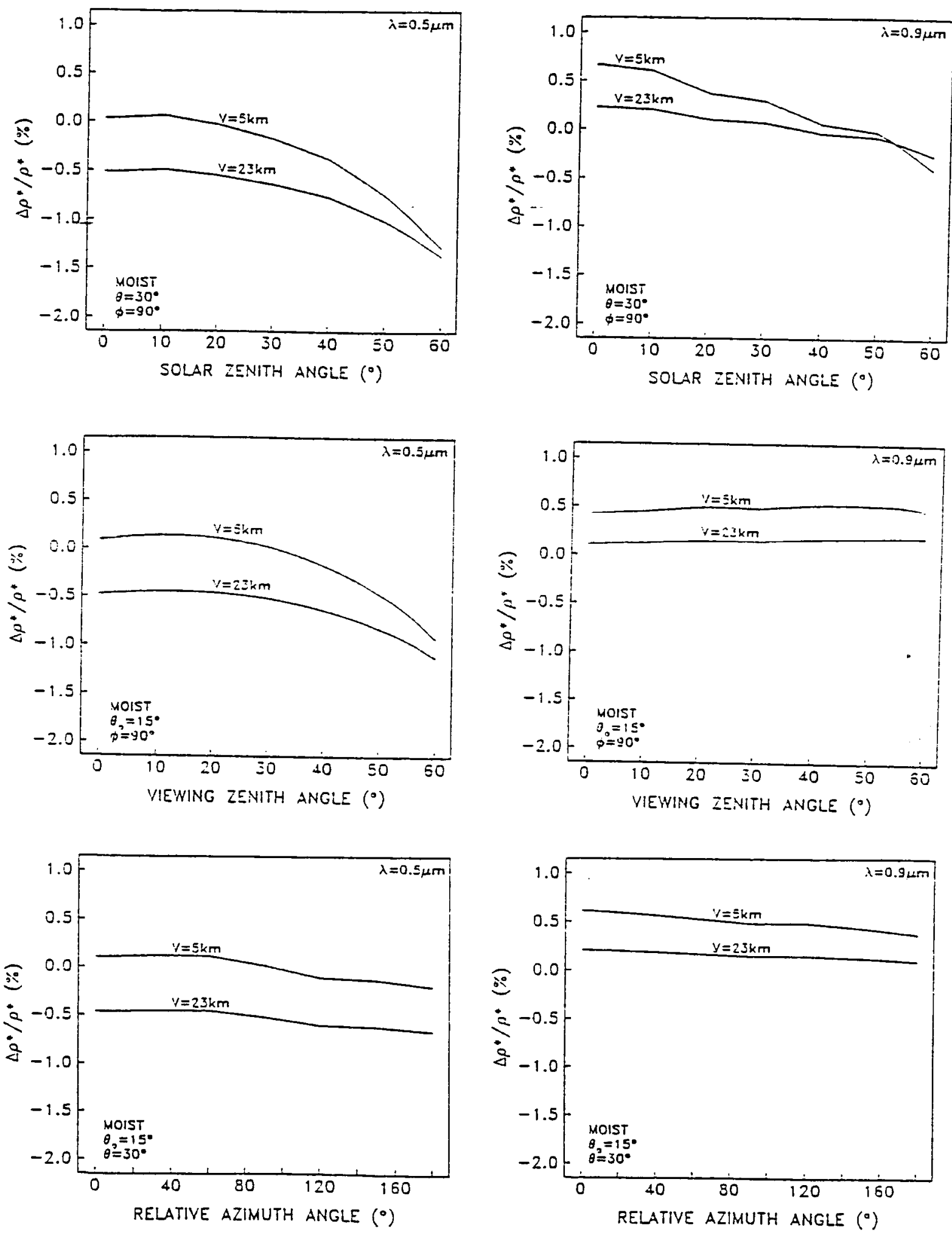

Fig. 11. Error introduced in the modeling of $\rho^{*}$ by replacing $\bar{\rho}$ and $\langle\rho\rangle$ with $\rho$. Positive values indicate underestimation. 


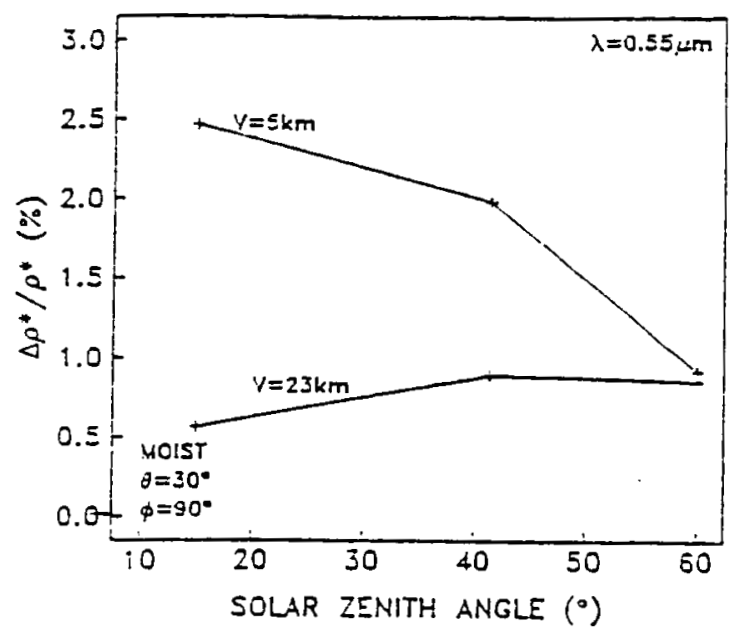

\section{ORIGNAL PAGE IS
OF POOP nilaLTY}
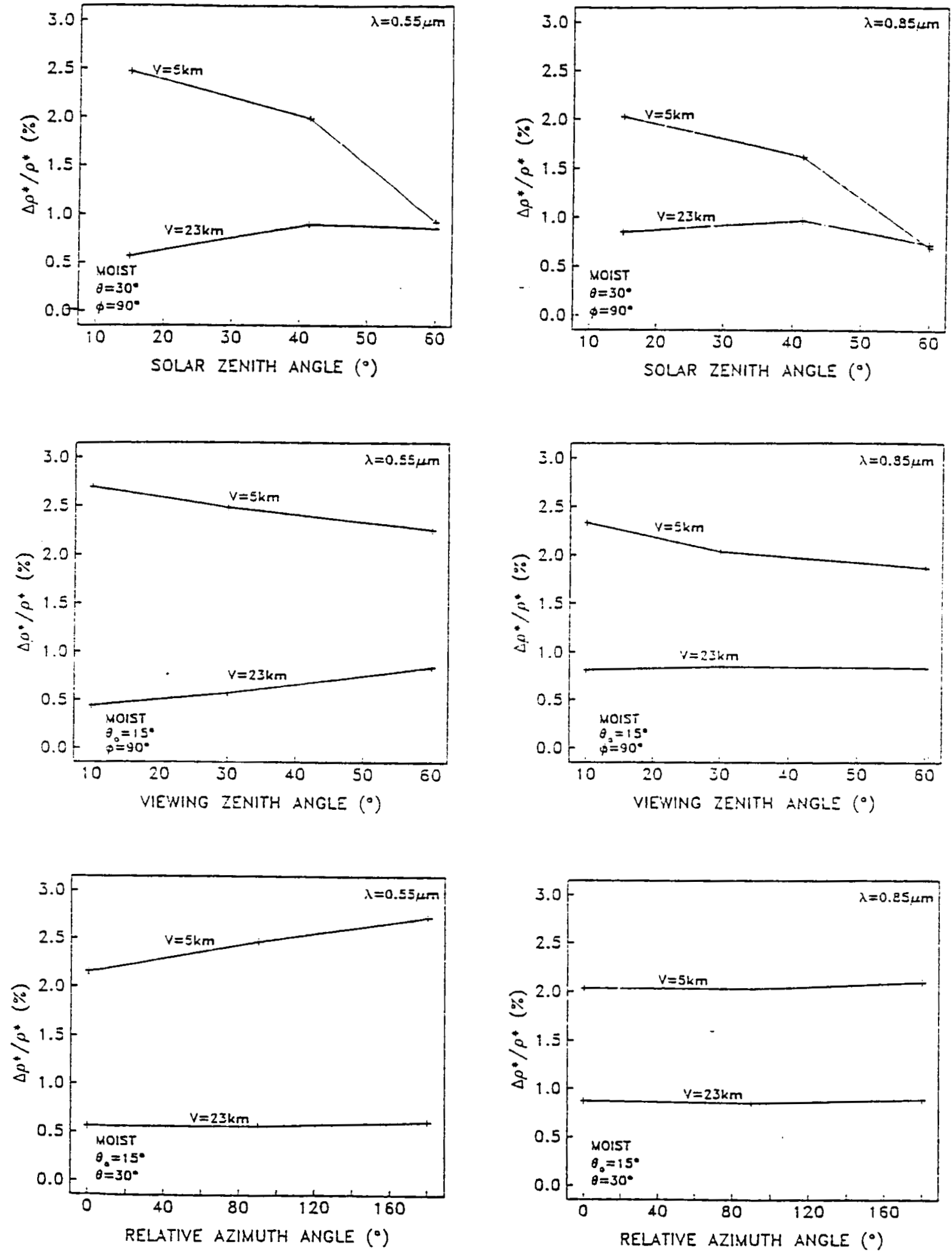

Fig. 12. Error in the modeling of $\rho^{*}$ in the case of Lambertian and homogeneous ground. The comparisons are made with exact calculations involving the successive orders of scattering method. Positive values indicate underestimation. 


\section{f. Accuracy}

In order to assess the accuracy of our modeling of the irradiance $E$ measured by the satellite-borne sensors, errors in the parameterization of gaseous absorption and on the model of Tanré et al., (1979) should be considered in addition of the errors that we just discussed above. According to Tanré et al., (1986), the error on $t_{g}$ is smaller than $1 \%$, except at grazing incidence and observations angles $\left(\theta_{0}, \theta>80^{\circ}\right)$. The model of Tanre et al. (1979) has not been validated against in situ measurements, even in the simple case of an homogeneous and Lambertian ground (a difficult task anyhow). Comparisons with exact calculations involving the successive order of scattering method indicate, however, that for turbid atmospheres with visibilities of about $23 \mathrm{~km}$, the model underestimates $\rho^{*}$ by less than $1 \%$ in the wavelength range of interest (Fig. 12). At $\lambda=0.5 \mu \mathrm{m}$, this error happens to partly compensate for the error introduced by our approximation of $\bar{\rho}$ and $\langle\rho\rangle$, but not at $\lambda=0.9 \mu \mathrm{m}$ (see above). In fact, by restricting our observations to reasonably clear conditions (visibility of $\sim 23 \mathrm{~km}$ ), the absolute value of the overall error expected on our modeling of $E$ should not exceed $2 \%$.

\section{Data}

\section{a. Satellite data sets}

The VISSR/VAS data used for the calibration were provided by the Space Science and Engineering Center of the University of Wisconsin, Madison in the form of full resolution (i.e., $\sim 1.5 \mathrm{~km}$ at the latitude of White Sands), 6-bit precision (i.e., 1/64) $256 \times 256$ pixel images coded on an 8-bit scale. The images were acquired at approximately 15 day intervals, and cover the periods from October 1983 through January 1985 for GOES-6 and from October 1983 through July 1984 for GOES-5 (i.e., until the failure of the satellite). For each selected day, the data set consists of three consecutive (i.e., half hourly separated) clear sky images of White Sands and one image of space, all taken near the local noon.

The NOAA-7 AVHRR data were chosen among orbit passes received in real time and archived at the Satellite Oceanography Facility of the Scripps Institution of Oceanography, San Diego. They also cover the period from October 1983 through January 1985. White Sands generally falls within the acquisition range of the ground station, but not always, and is often located near the east edge of the $2900 \mathrm{~km}$-wide swath. The passage over the antenna site occurs at about 16:00 local time, which is relatively late in the afternoon and, 


\section{ORIGINAL PAGE IS \\ OF POOR QUALTY}

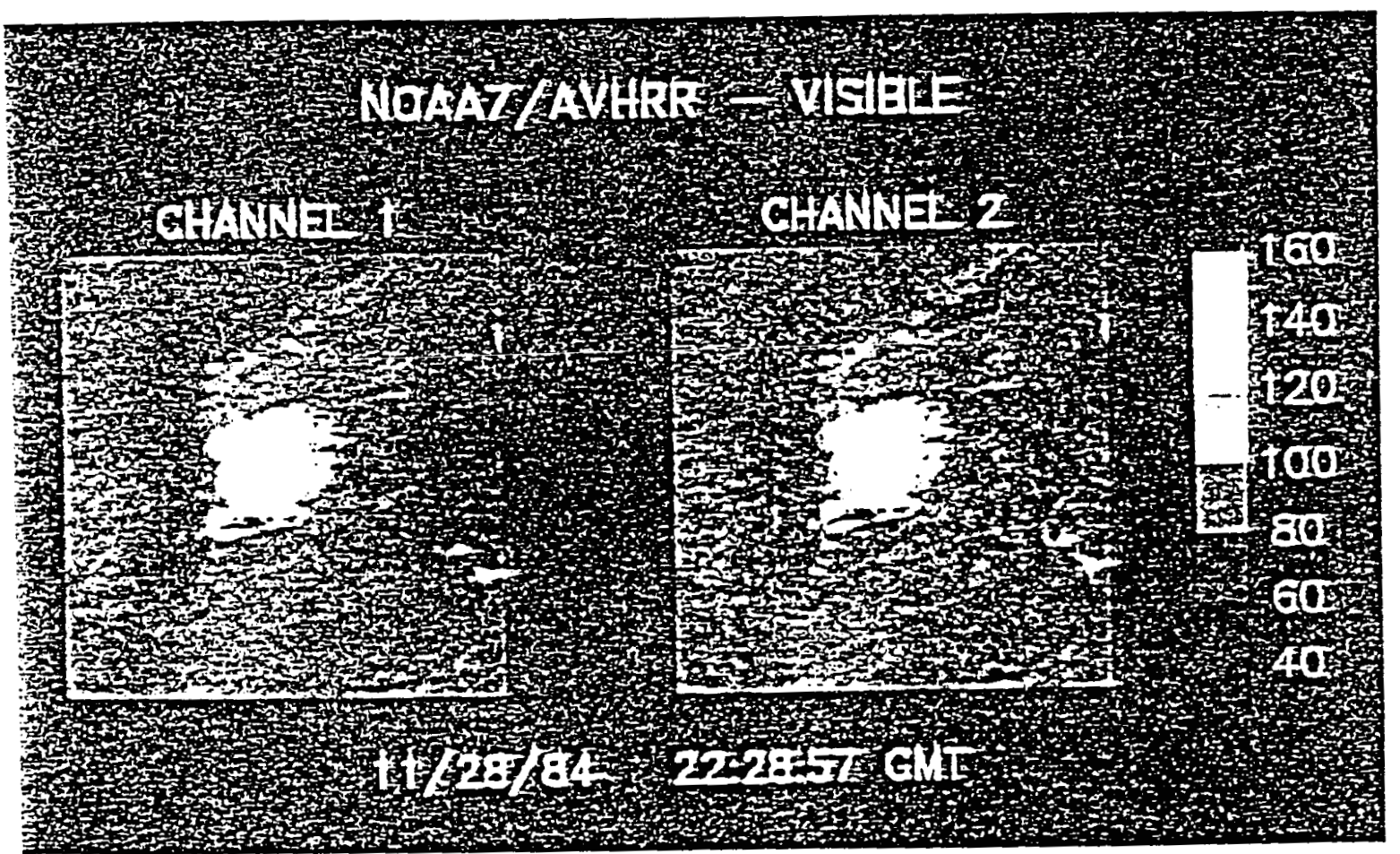

Fig. 13. Examples of White Sands images in the AVHRR solar channels. 


\section{ORIGINAL PAGE IS \\ OF POOR QUALTTY}

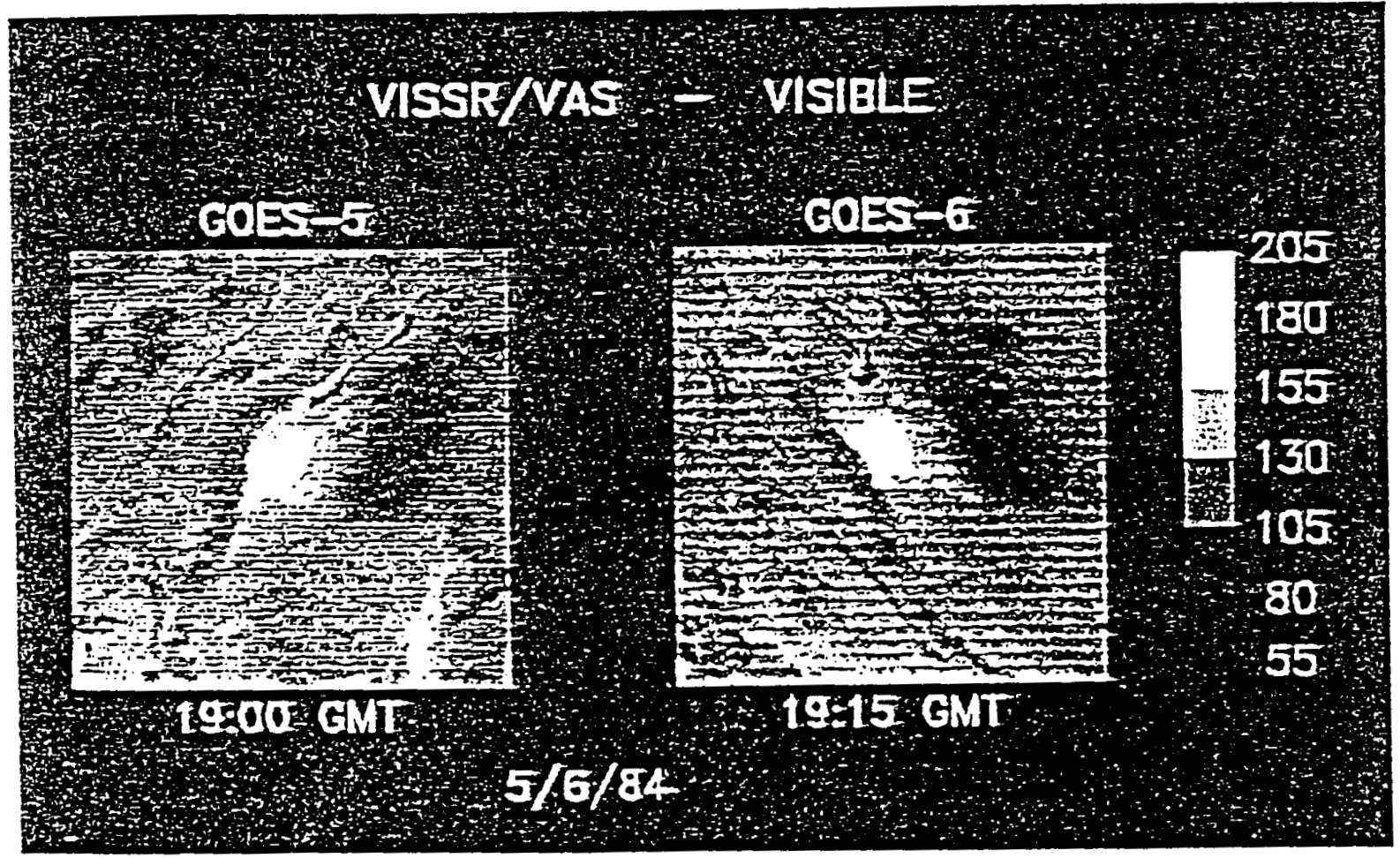

Fig. 14. Same as Fig. 13, but VISSR/VAS instead of AVHRR. 
consequently, not optimum for the calibration, especially during winter (too low sun above the horizon). The direct readout High Resolution Picture Transmission data telemetered to the station are 10-bit in precision (i.e., 1/1024) and the ground resolution at nadir is $1.1 \mathrm{~km}$.

Figs. 13 and 14 give examples of White Sands images in the VISSR/VAS and AVHRR shortwavelength channels, respectively. White Sands is easily distinguishable from the surrounding environment by its high brightness (white tones). The shape of the area appears somewhat distorted from one image to the other, a direct consequence of the different viewing geometries. The demarcation between the flats and the more reflective dunes, not discernible on the VISSR/VAS images (Fig. 13) is clearly apparent on the AVHRR images (Fig. 14). The contrast, in fact, depends on the geometrical conditions of observation. The VISSR/VAS images exhibit stripes, which illustrate the differences among the detectors (see section 2). This suggests that the destriping procedure applied by NOAA CDA might not be completely efficient, since the corresponding space images (not shown here) were relatively free of stripes.

Sub-images of the White Sands dunes were extracted from the original satellite data sets. Since no geometric corrections were applied to the data, the number of pixels contained in the sub-images is not constant, but depends on the viewing geometry (we seek to include as many pixels as possible to improve the statistics of the calibration results). Since our objective for the VISSR/VAS is to perform an average calibration of the eight shortwavelength detectors, eight consecutive lines form the sub-images. The space counts at the beginning of the AVHRR scan lines that correspond to the White Sands viewing and the entire $256 \times 256$ pixel VISSR/VAS images of space constitute the zero intensity data set used in the computations.

Average White Sands and space counts and their respective standard deviations are presented in Figs. 15 and 16 for AVHRR and VISSR/VAS shortwavelength channels, respectively. Over the study period, the average space count is practically constant for AVHRR channels 1 and 2, and does not significantly change for the VISSR/VAS shortwavelength channels. In particular, no appreciable shift or trend can be detected. The average White Sands count, on the contrary, strongly varies with time. The low frequency flucuations reflect those of the solar radiance at the top of the atmosphere since, for each satellite, the observations were taken at approximately the same time during the entire study period. In some instances, abrupt changes are observed at about two week intervals 

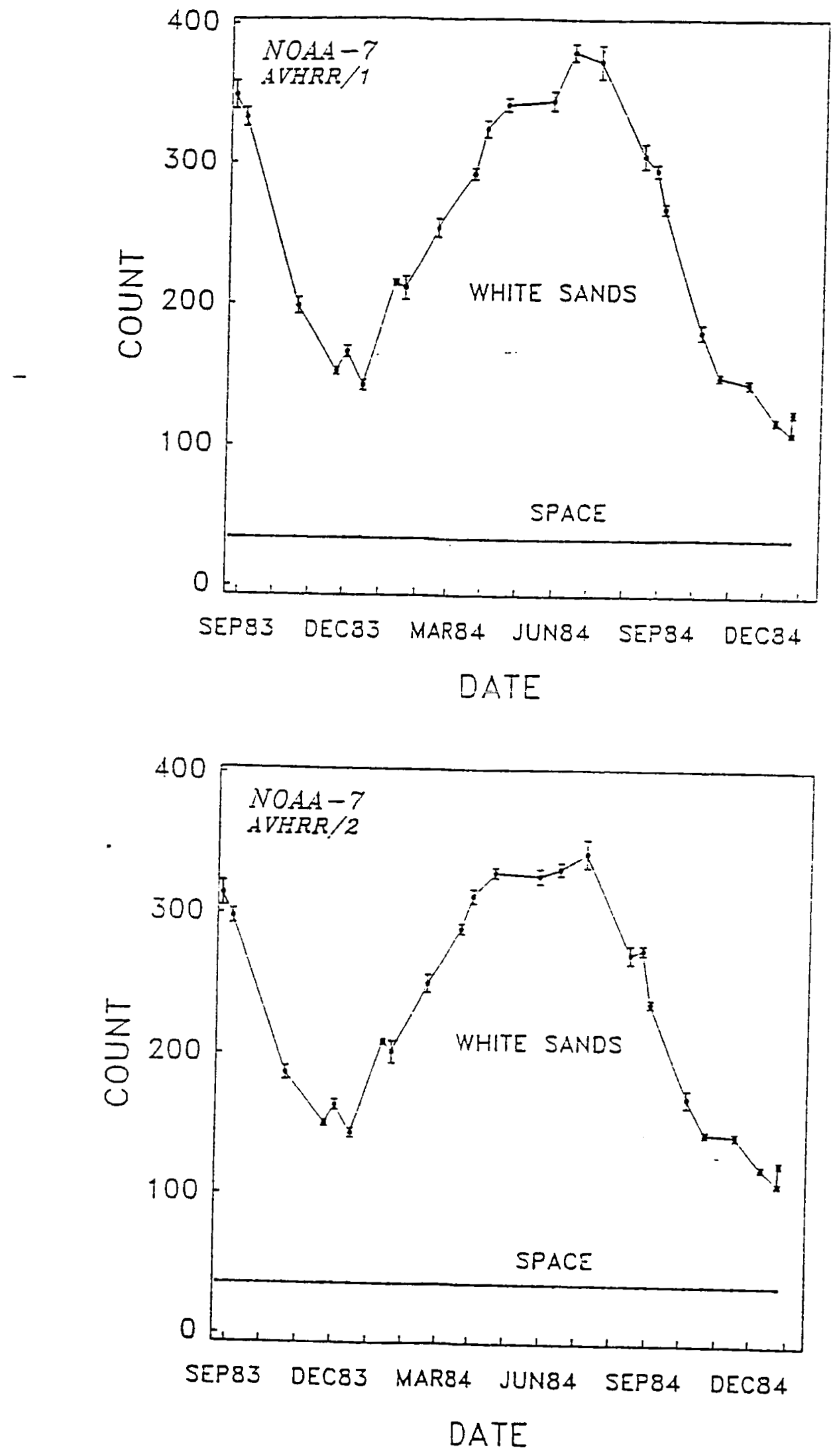

Fig. 15. White Sands and space AVHRR brightness counts for the study period. 


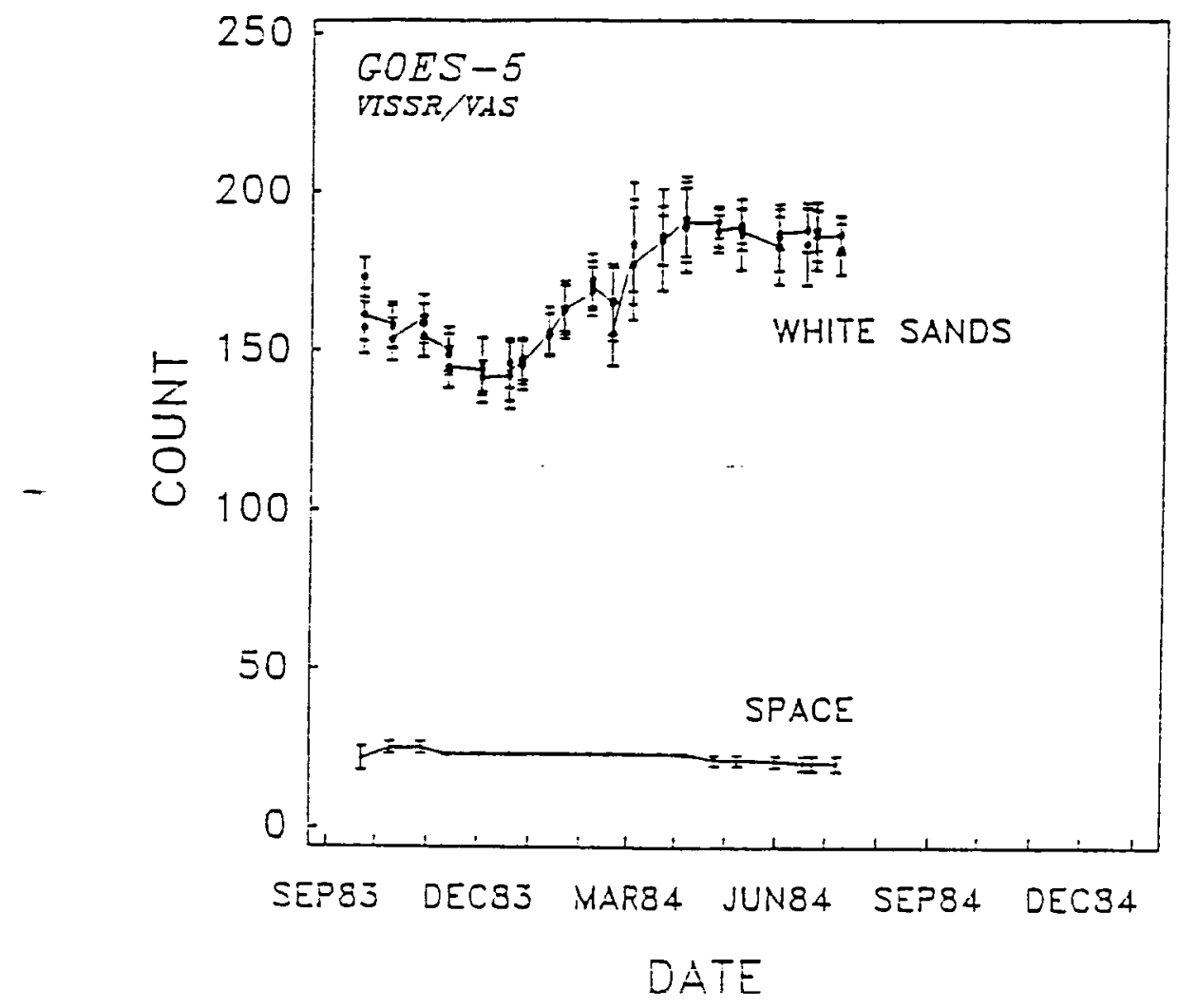

ORIGINAL PAG: IS OF POOR QUALTY

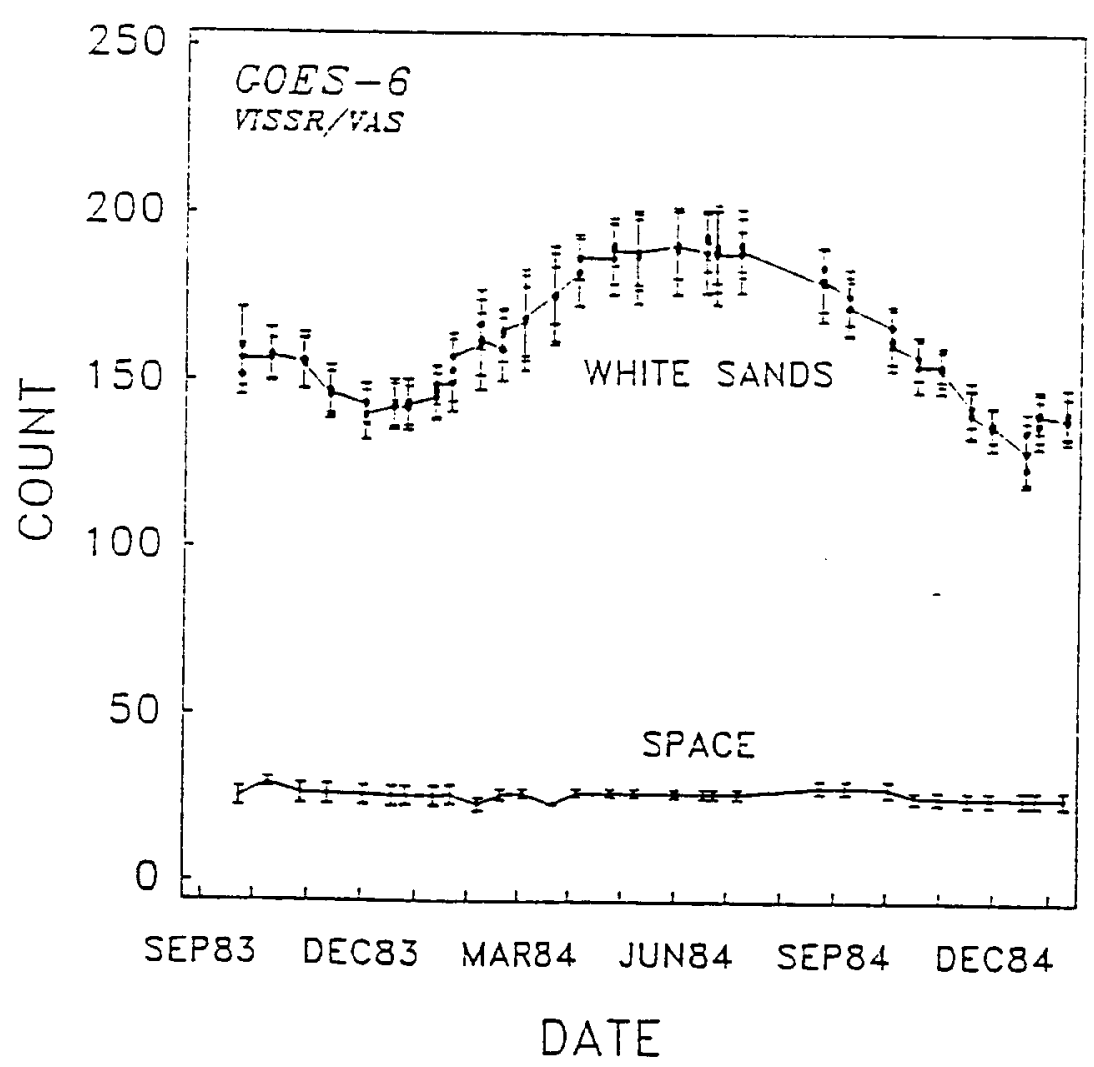

Fig. 16. Same as Fig. 15, but VISSR/VAS instead of AVHRR. 
(e.g., between February 2 and February 8, 1984 for GOES-5) or from one image to the next during the same day (e.g., on December 30, 1984 for GOES-6). The spatial (i.e., horizontal) standard deviation is generally small (2 to 6 counts) for AVHRR channels 1 and 2, but often exceeds 10 counts for the VISSR/VAS shortwavelength channels, and may result from surface moisture variations, inhomogeneities in the surface composition, local changes in the characteristics of the atmosphere, intrinsic instrumental noise, differences among the detectors (for GOES-5 and GOES-6) and instrument changes.

\section{b. Viewing geometry}

The angles characterizing the incidence and observation directions are displayed in Figs. 17, 18 and 19 for GOES-5, GOES-6, and NOAA-7, respectively.

During the study period, the solar zenith angle varied from 10 to $60^{\circ}$ for GOES-5 and GOES-6, and from 45 to $80^{\circ}$ for NOAA-7. The low frequency fluctuations exhibit a sinusoidal form, which is intimately related to the annual cycle of the sun. The NOAA-7 solar zenith angles are generally large, which makes their selection for an accurate calibration questionable. But we are limited to the afternoon NOAA-7 passes, and they occur when the sun is already low.

The viewing zenith angles from the geostationary altitude remain fairly constant (i.e., $\sim 50^{\circ}$ for GOES-5 and $\sim 49^{\circ}$ for GOES-6) until GOES-5 failed in July 1984 . GOES-6, stationed over the Equatorial Pacific at $135^{\circ} \mathrm{W}$ at the moment of the failure, was then maneuvered over the United States to the longitude of $85^{\circ} \mathrm{W}$, and the viewing zenith angle changed to about $38^{\circ}$. The NOAA-7 viewing zenith angle is subjected to more important, somewhat erratic changes (Fig. 17), which in fact depend on the distance of the satellite subtrack to White Sands (the closer the satellite passes the area, the smaller is the viewing angle).

Successful capture by the tracking antenna generally implies that NOAA-7 pass westward of the target. Since, on the other hand, the data are acquired when the sun is in the western quadrant, the sun and the satellite are viewed by the target from about the same azimuthal direction, thereby leading to small relative azimuth angles. On one occasion (i.e., November 30, 1984), however, the satellite passed close to, but east of White Sands and a relative azimuth angle of nearly $158^{\circ}$ was observed. For GOES-5 and GOES-6, the relative azimuth angle rapidly varies from one hour to the next (Figs. 18 and 19). This is simply due to the fact that the observations are taken near the local noon, i.e., near the 
ORIGINAL PRGR IS

OF POOR QUALTY
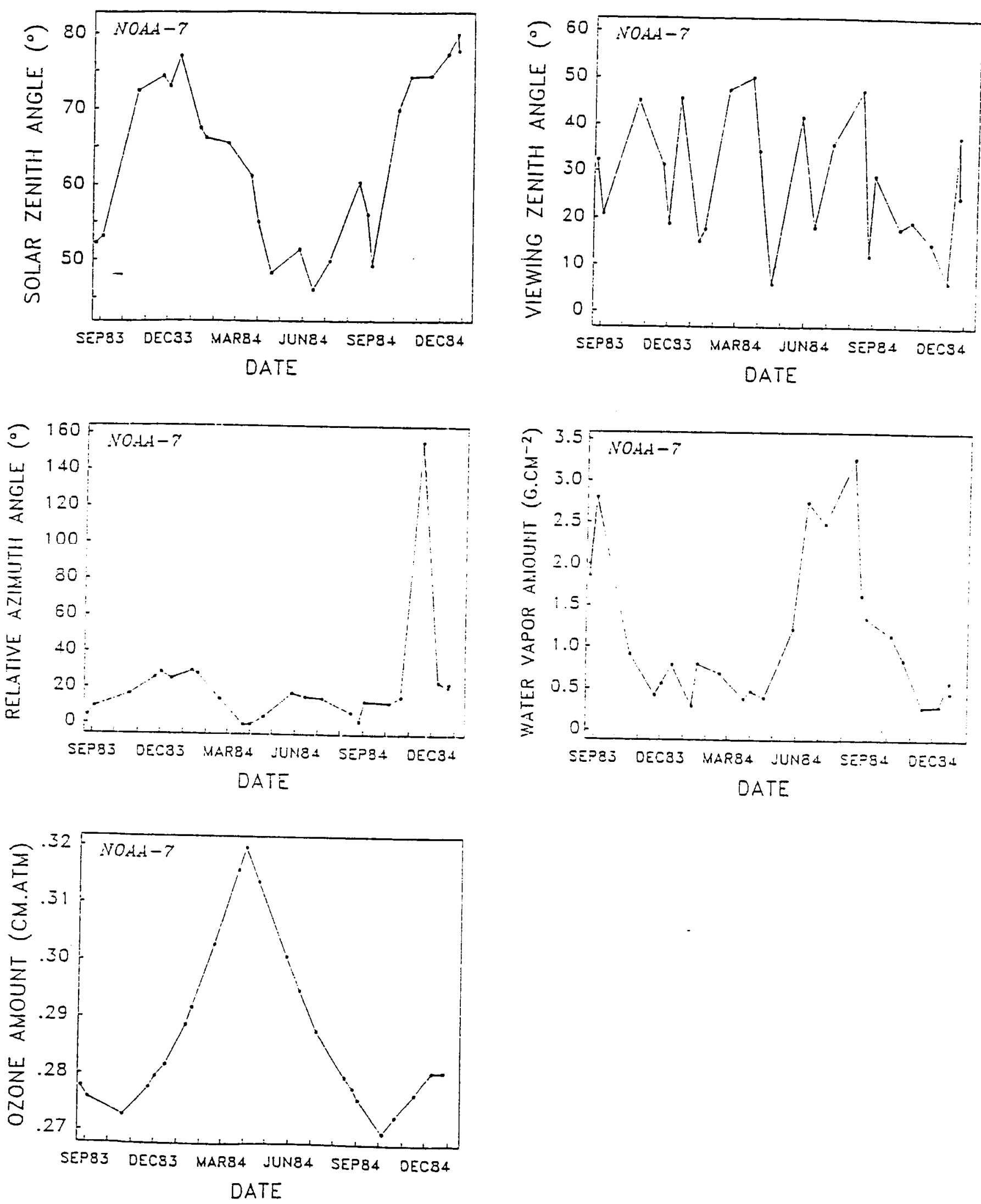

Fig. 17. NOAA-7 viewing geometry and atmospheric characteristics at the moment of satellite overpass. 

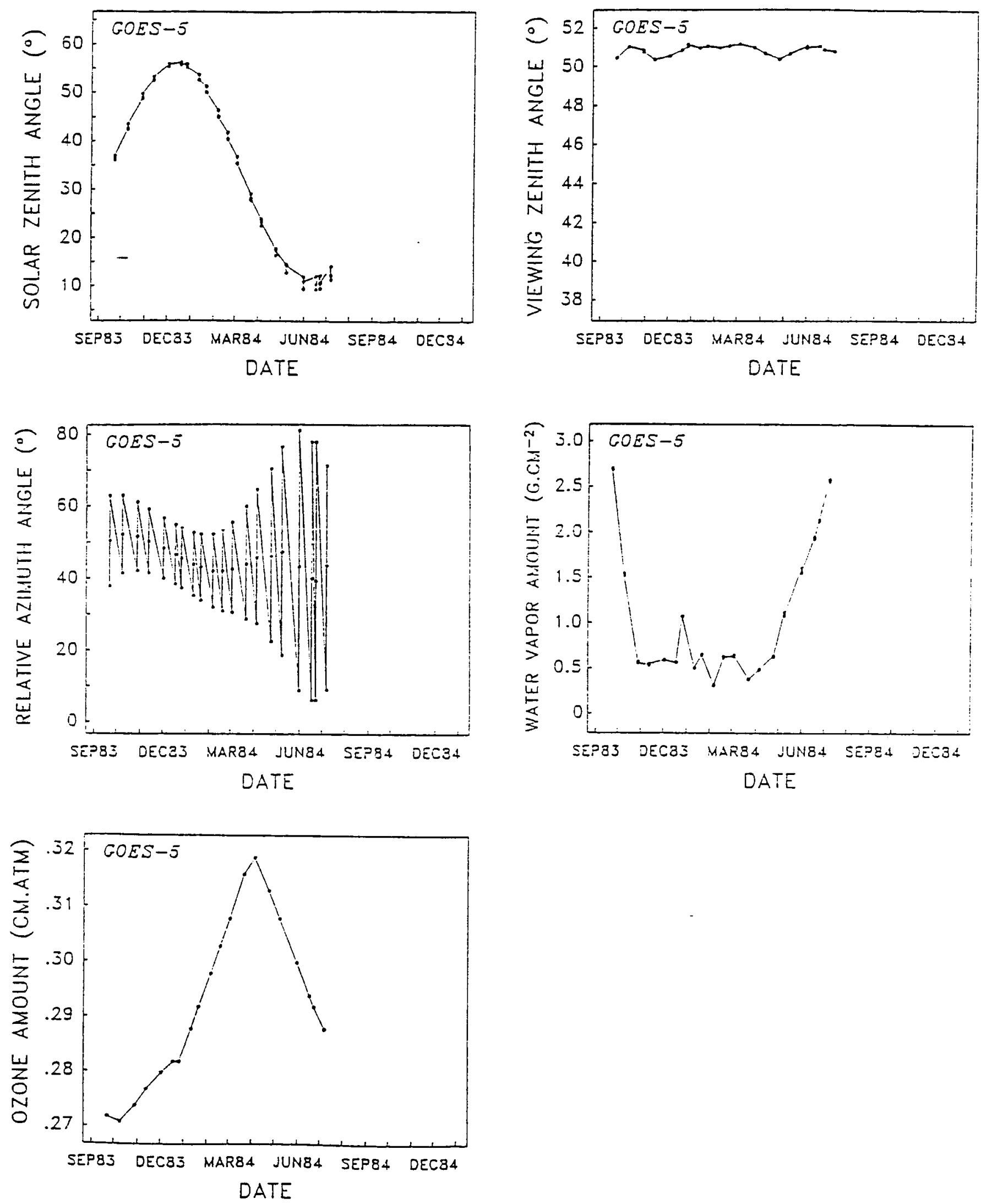

Fig. 18. Same as Fig. 17, but GOES-5 instead of NOAA-7. 

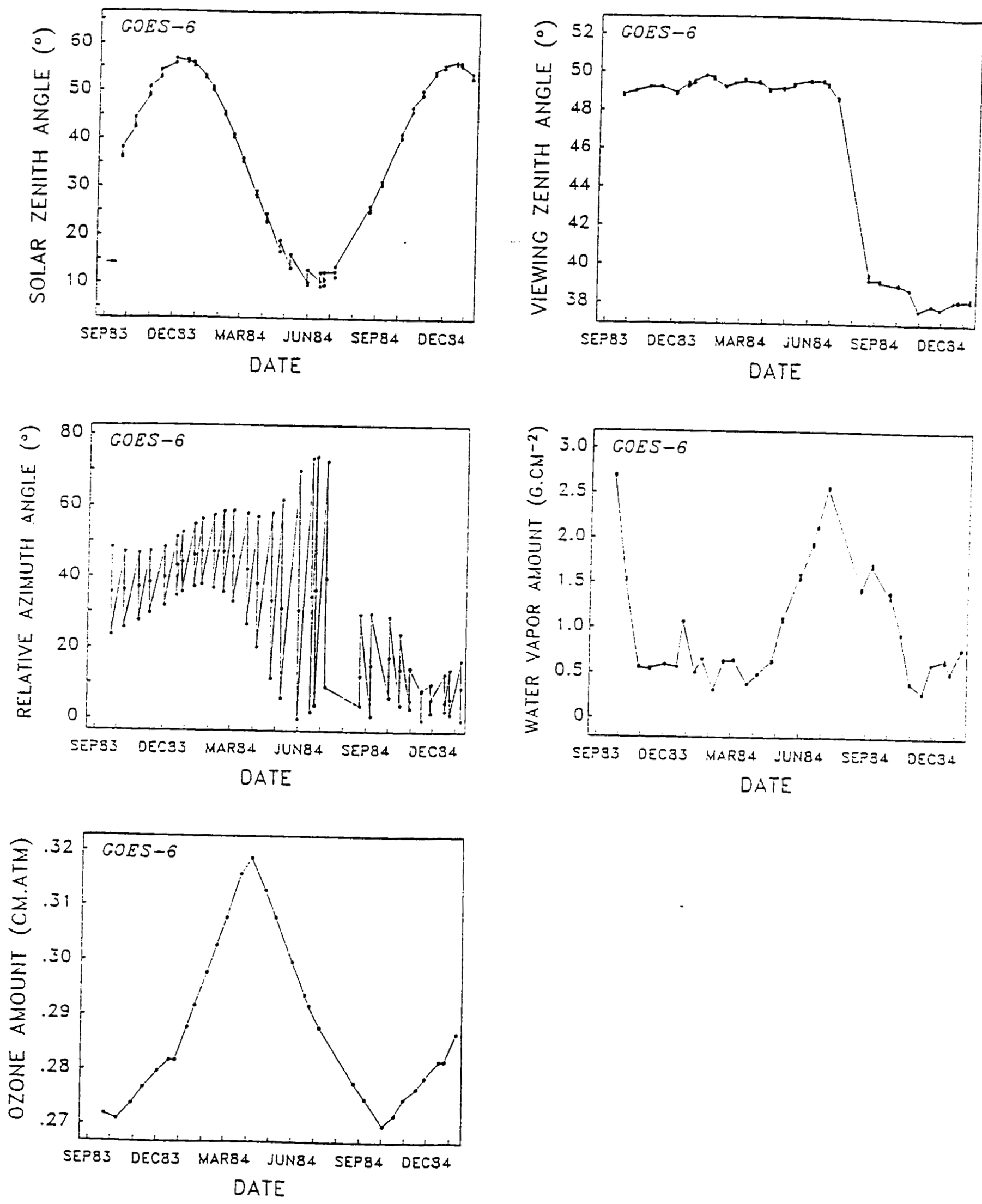

Fig. 19. Same as Fig. 17, but GOES-6 instead of NOAA-7. 
maximum solar elevation during the day. The amplitude of the fluctuations is larger during summer, because the sun is higher at noon during that season. Note also in Figs. 18 and 19 that before the failure of GOES-5, the GOES-5 and GOES-6 relative azimuth angles vary in opposite direction during the day. The reason for this is that the two satellites were viewing White Sands from directions located respectively east and west of the target. Indeed, after the failure of GOES-5, the same type of variations as those observed for GOES-5 are encountered for GOES-6, except that the magnitude of the relative azimuth angle is smaller, a consequence of the larger GOES-6 azimuth angle (the GOES-6 subpoint on the earth is closer to the White Sands meridian).

\section{c. Meteorological parameters}

The vertically integrated ozone amounts were estimated from the meteorological atlas of London et al., (1976). At the latitude of White Sands, the values range from $0.27 \mathrm{~cm}$.atm in autumn to $0.32 \mathrm{~cm} . \mathrm{atm}$ in spring. The vertically integrated water vapor amounts were obtained from radiosonde observations at El Paso $\left(31^{\circ} 48^{\prime} \mathrm{N}, 106^{\circ} 24^{\prime} \mathrm{W}\right)$, a meteorological station located at nearly the same altitude and longitude as White Sands. No corrections for the latitudinal distance between El Paso and White Sands (about $120 \mathrm{~km}$ ) were effected. This procedure is roughly correct because the atmospheric perturbances usually travel west in that region. The atmospheric visibility is that visually observed at meteorological station HMN, a station very close (a few $\mathrm{km}$ ) to the calibration site. Unfortunately, a standard value of $25 \mathrm{~km}$ was reported continuously during the entire study period. The actual visibility might in fact be much larger in many cases: values of $100 \mathrm{~km}$ or more have been frequently observed (Slater, personal communication). Our deficient knowledge of the visibility at White Sands is indeed a potential source of error for the calibration, which can be estimated (see below) but not accounted for. At White Sands, the atmospheric pressure is considerably reduced because of the surface altitude. This was carefully accounted for in the computations of the Rayleigh optical thickness. Atmospheric pressure also affects the absorption strength of the intervening gases as in the case of temperature, but the error that is introduced when using the US62 standard profiles of pressure and temperature is neglected because of its secondary importance.

\section{Results}

The satellite irradiances computed for White Sands, with the data and the model discussed above, are presented in Fig. 20 for NOAA-7 and in Fig. 21 for GOES-5 and 

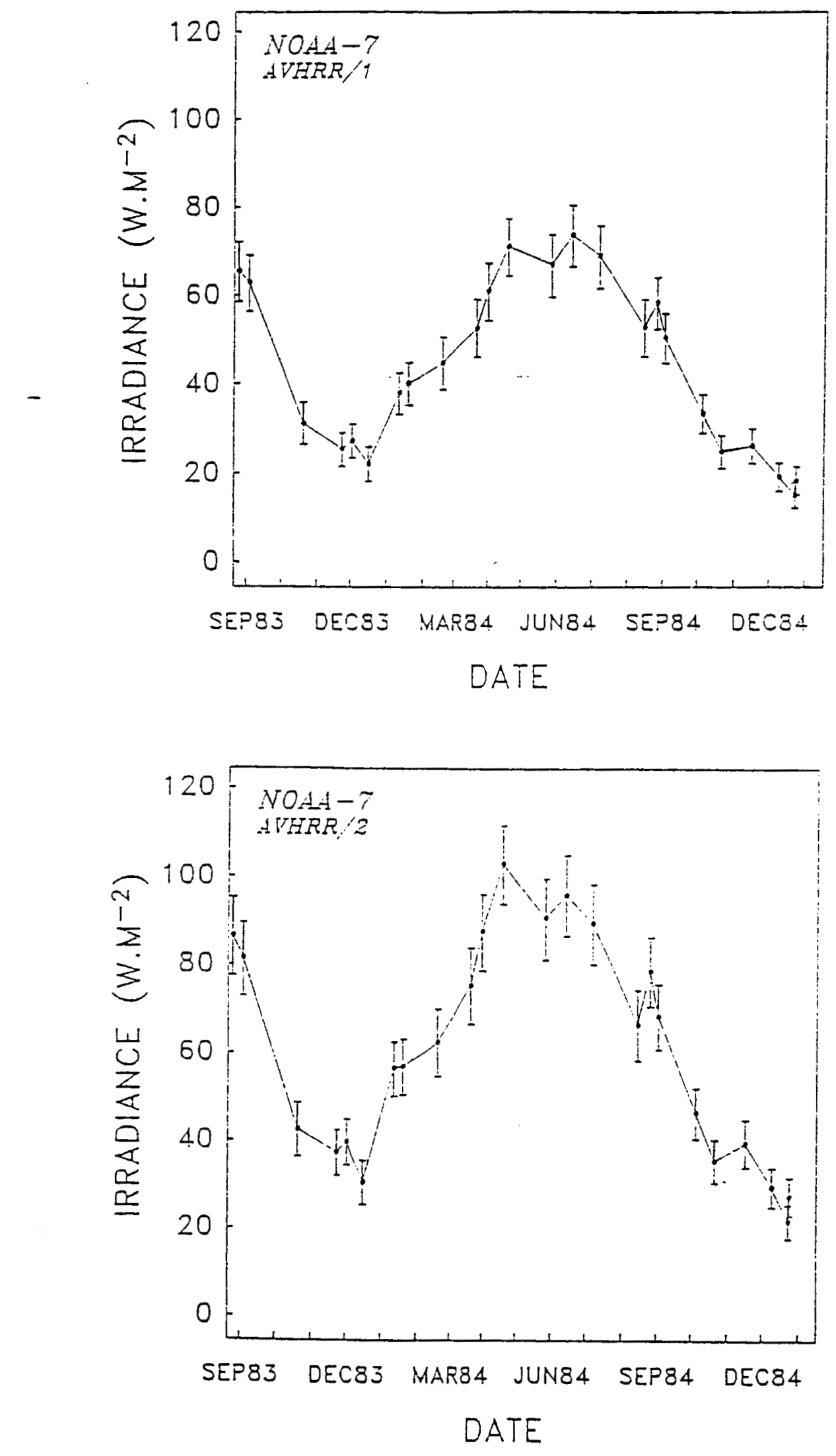

Fig. 20. Modeled White Sands AVHRR irradiance for the study period. 

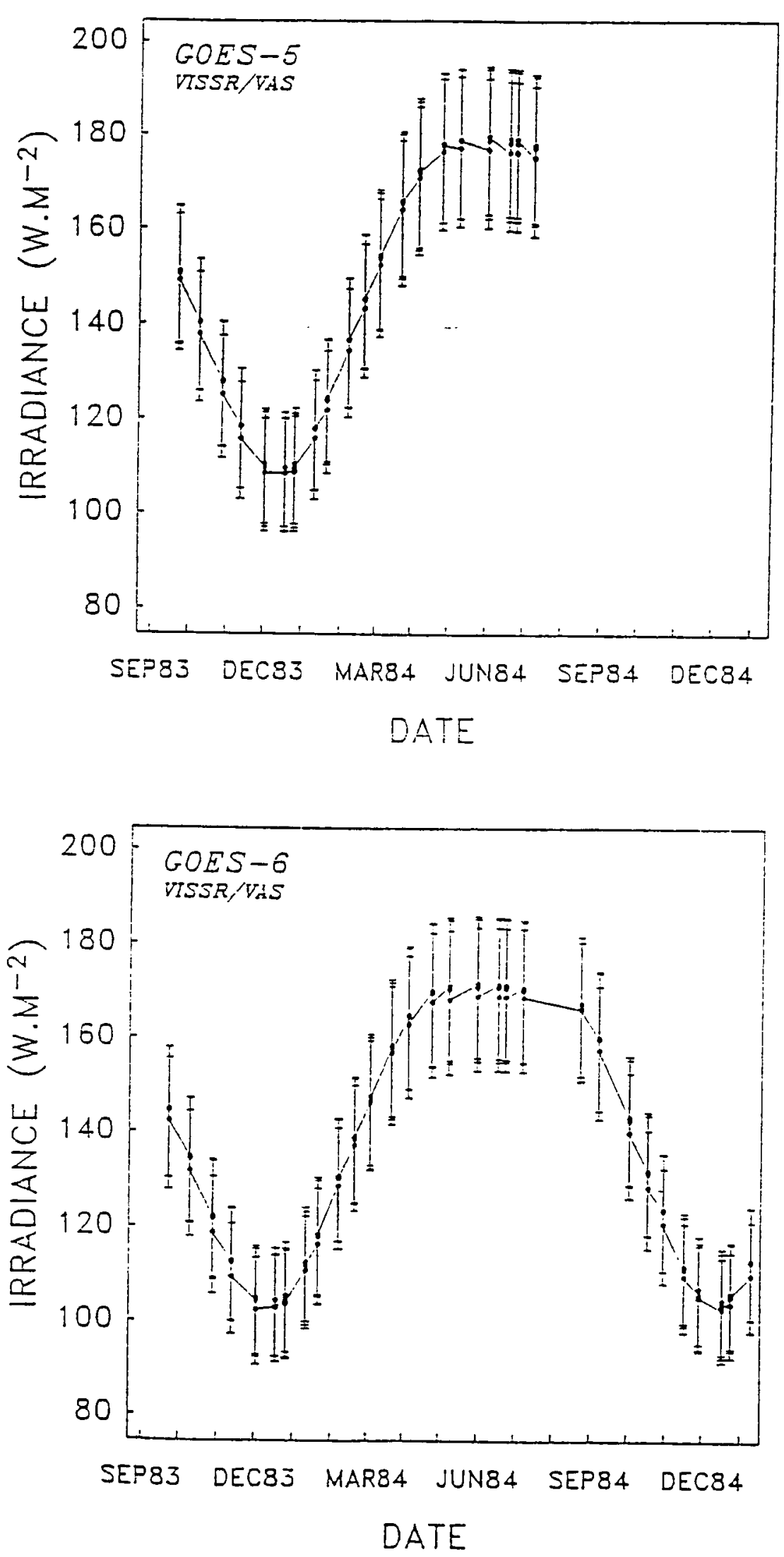

Fig. 21. Same as Fig. 20, but VISSR/VAS instead of AVHRR. 
GOES-6. They correspond to the brightness counts of Figs. 15 and 16, respectively. Large variations on the seasonal time scale are clearly present in Figs. 20 and 21, of the order of 80 and $60 \mathrm{Wm}^{-2}$ for AVHRR channels 1 and 2, respectively, and of $80 \mathrm{Wm}^{-2}$ for the VISSR/VAS visible channels. For consecutive times during a given day, the VISSR/VAS irradiances vary by only a few $\mathrm{Wm}^{-2}$ because the sun zenith angle does not change much during the one-and-one-half hour period of the observations.

The modeled irradiances fairly well reproduce the seasonal or even monthly variations of the counts, but not those existing at smaller time scales, especially from one hour to the next in the case of GOES-5 and GOES-6. This is partly due to our method of specifying the atmospheric parameters, which does not account for changes occurring at hourly time scales. Also, the short-term variations of the counts may result from instrument modifications, which indeed are not considered in the method.

To evaluate the accuracy of the simulated irradiances, uncertainties of $30 \%$ on the anisotropy factor of the aerosol phase function, $30 \%$ on the atmospheric visibility, $10 \%$ on the ozone amount, $20 \%$ on the water vapor amount, $50 \%$ on the soil humidity and $1 \%$ on the surface reflectance (due to other processes than soil humidity) were assumed. Uncertainties on other aerosol parameters, such as their vertical distribution, are not significant to the first order. The $10 \%$ uncertainty on the ozone amount corresponds to the daily fluctuations around the geographical monthly mean (London et al., 1976). The $20 \%$ uncertainty on the water vapor amount is typically that of radiosonde observations. The $50 \%$ uncertainty on the soil humidity is representative of the annual variations reported by Williamson (1975). The $1 \%$ uncertainty on the surface reflectance accounts for errors in the measurements of Walraven and Coulson (1972). The 30\% uncertainty in the asymmetry parameter is characteristic of differences between the extreme cases of maritime and urban aerosols. Finally, the $30 \%$ uncertainty on the atmospheric visibility is somewhat arbitrary, because the visibility at the calibration site is poorly known. But the major problem with the visibility undoubtedly arises from the standard value of $25 \mathrm{~km}$ consistently reported by meteorological station HMN. Is this value representative of the average conditions at the calibration site? If not, and recent observations tend to indicate that the visibility at White Sands is often larger $(>100 \mathrm{~km})$, the modeled irradiances are consequently biased (i.e., underestimated) by several percents, more precisely by $2.5 \%$ if the average visibility is $100 \mathrm{~km}$. Thus, except for the visibility, the uncertainties estimated for the atmospheric and surface parameters are reasonable, and would most likely maximize the overall error 
Table 2. Uncertainties on model parameters and corresponding errors in simulated irradiances.

\begin{tabular}{|c|c|c|c|c|}
\hline \multirow[b]{2}{*}{$\mathbf{P}$} & \multirow{2}{*}{$\begin{array}{c}\Delta P / P \\
(\%)\end{array}$} & \multicolumn{3}{|c|}{$\begin{array}{c}\Delta E / E \\
(\%)\end{array}$} \\
\hline & & VISSR/VAS & AVHRR/1 & AVHRR/2 \\
\hline $\begin{array}{l}\text { Anisotropy Factor } \\
\text { Visibility }\end{array}$ & $\begin{array}{l}30 \\
30\end{array}$ & $\begin{array}{l}0.5 \\
0.9\end{array}$ & $\begin{array}{l}0.7 \\
1.1\end{array}$ & $\begin{array}{l}0.6 \\
1.0\end{array}$ \\
\hline $\begin{array}{l}\text { Water Vapor Amount } \\
\text { Ozone Amount }\end{array}$ & $\begin{array}{l}20 \\
10\end{array}$ & $\begin{array}{l}0.2 \\
0.6\end{array}$ & $\begin{array}{l}0.2 \\
0.7\end{array}$ & $\begin{array}{r}1.2 \\
<0.1\end{array}$ \\
\hline $\begin{array}{l}\text { Surface Reflectance } \\
\text { Soil Humidity }\end{array}$ & $\begin{array}{r}1 \\
50\end{array}$ & $\begin{array}{l}1.5 \\
2.2\end{array}$ & $\begin{array}{l}1.4 \\
1.9\end{array}$ & $\begin{array}{l}1.3 \\
2.1\end{array}$ \\
\hline
\end{tabular}


on the modeled irradiances. On top of these uncertainties, one should not forget the uncertainty on the radiative tranfer model itself (about $2 \%$ for most situations encountered in the study; see Section 3e). When introduced together in the model, the uncertainties discussed above yield the errors shown in Figs. 20 and 21, computed for all individual cases. These errors range from 8 to $13 \%$, and might explain the inability of the method to retrieve the short-term variability of the brightness counts.

To apprehend what are the most critical parameters for the calibration, the different uncertainties were considered separately around average conditions for the atmosphere. The results are presented in Table 2. Clearly, the most contributing errors are those on the soil humidity, the surface reflectance and on the atmospheric visibility. The influence of the anisotropy factor is relatively limited, because the effects of this parameter on $\rho_{a}$ on the one hand and on $A, B$ and $C$ on the other hand (see Eq. 5) partly compensate. For the ozone amount, our treatment based on climatological data appears to be sufficient. For the water vapor amount, if the utilization of radiosonde observations is satisfactory for the VISSR/VAS visible channels and for AVHRR channel 1, it yields an error of about 1.2\% for AVHRR channel 2. This error, as most of the other errors, can be reduced, for instance by using optical instruments on site (e.g., pyrheliometer for the turbidity, Voltz photometer for the water vapor amount). This would require, however, increased means and effort, and will be discussed in the final section as part of a global strategy to control the quality of the data acquired in orbit.

From the White Sands and space average counts (Figs. 15 and 16), the modeled irradiances (Figs. 20 and 21), and assuming that the space irradiance is null, average calibration coefficients were calculated. This was done by comparing the modeled irradiances with the corresponding AVHRR 10-bit counts (Fig. 22) or VISSR/VAS 8-bit counts squared (Fig. 23). The correlation coefficient is better than 0.995 at the $99 \%$ confidence level for both types of instrument. Assuming that the calibration points should fit a straight line of the form (1) or (2), the best estimates of the slope and intercept were computed by means of the principle of maximum likelihood, and are given in Table 3. In the computations, however, the linear regressions were forced toward the space points, because the irradiance of space (equal to zero) is not subjected to uncertainty and, further, the time variability of the space counts is small. If this constraint were not introduced, the results would have changed significantly for GOES-5 and GOES-6 because the VISSR/VAS calibration points are not well distributed, yielding less accurate calibration coefficients. The residual errors 

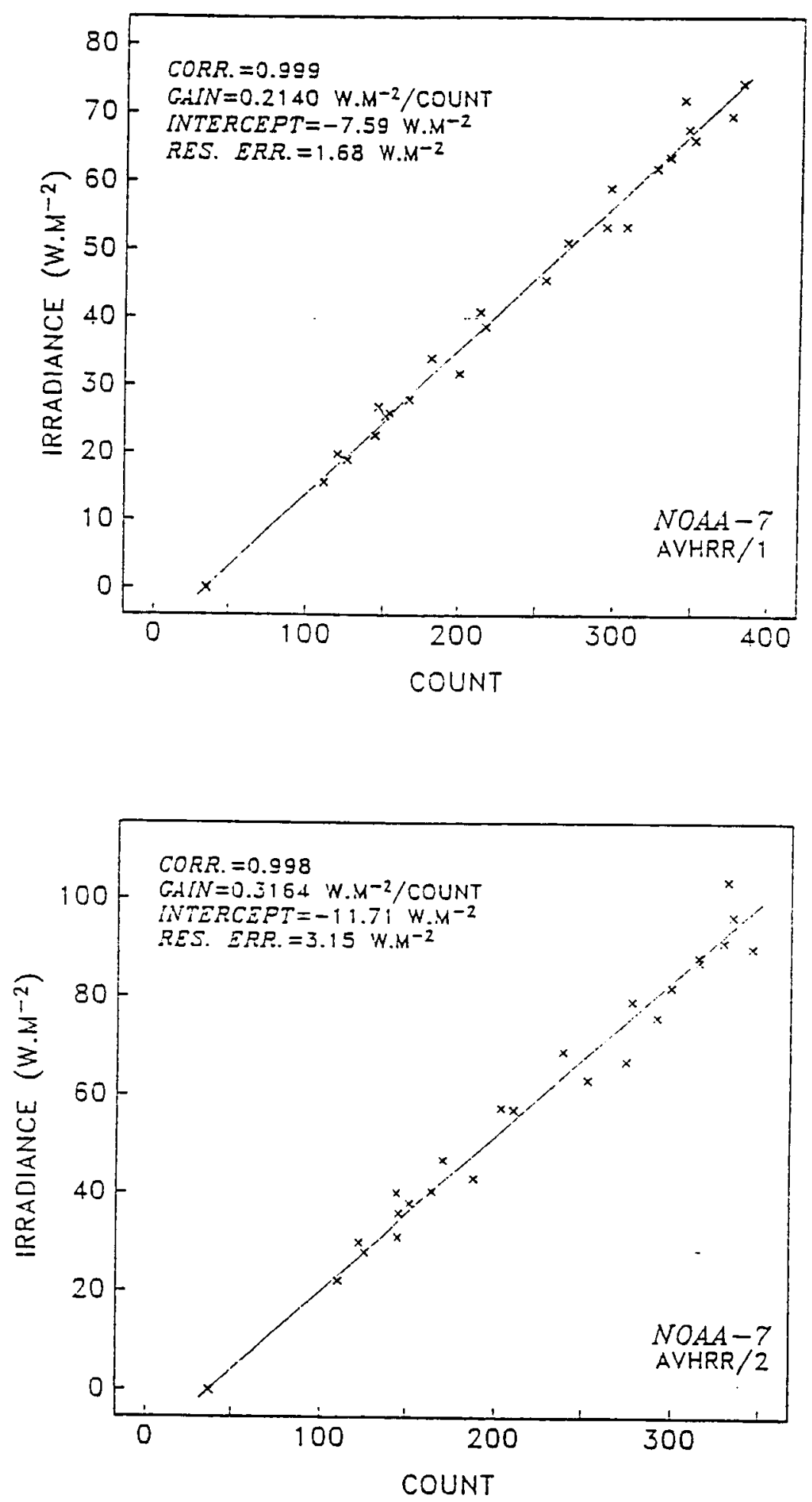

Fig. 22. Plots of modeled White Sands AVHRR irradiance versus brightness counts. Statistics of best linear fits are specified on the figure. 
ORIGINAI RAEE G.

OF POOR QUALTY
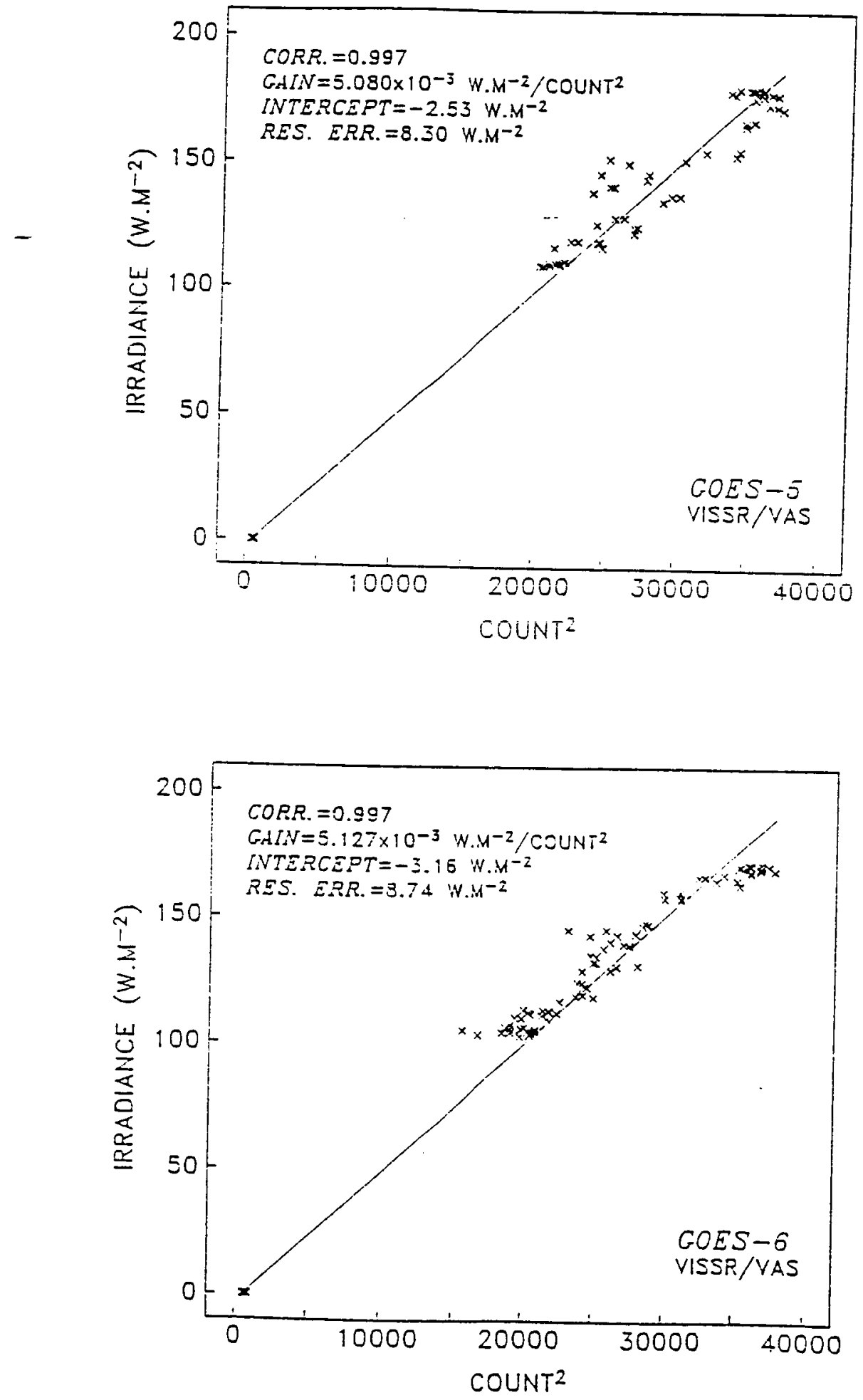

Fig. 23. Same as Fig. 22, but VISSR/VAS instead of AVHRR. 
Table 3. Average post-launch calibration coefficients.

\begin{tabular}{lcc}
\hline \multicolumn{1}{c}{ Satellite/Sensor } & $\begin{array}{c}\text { Gain } \\
\left(\mathrm{Wm}^{-2} / \text { count }\right. \\
\left.\text { or } \mathrm{Wm}^{-2} / \text { count }^{2} \ddagger\right)\end{array}$ & $\begin{array}{c}\text { Intercept } \\
\mathrm{Wm}^{-2}\end{array}$ \\
\hline GOES-5 VISSR/VAS & $5.080 \times 10^{-3} \pm 0.069 \times 10^{-3}$ & $-2.5 \pm 1.8$ \\
GOES-6 VISSR/VAS & $5.127 \times 10^{-3} \pm 0.064 \times 10^{-3}$ & $-3.2 \pm 1.5$ \\
NOAA-7 AVHRR/1 & $0.2137 \pm 0.0020$ & $-7.5 \pm 0.4$ \\
NOAA-7 AVHRR/2 & $0.3154 \pm 0.0041$ & $-11.5 \pm 0.7$ \\
\hline †AVHRR & & \\
†VISSR/VAS & &
\end{tabular}


are small, i.e., about $5 \%$ of the mean irradiance value for the VISSR/VAS visible channels (Fig. 22), and only $3 \%$ and $4 \%$ respectively for AVHRR channels 1 and 2 (Fig. 23). This provides a good idea of the level of stability of the calibration coefficients, and tends to indicate that our assessment of the errors on the modeled irradiances is pessimistic, but the residual errors do not include eventual biases.

\section{Discussion}

One of the goals of the calibration is to assess whether the instruments are experiencing changes (e.g., drift) as a result of performance degradation in flight. This can be verified by examining the evolution with time of the calibration coefficients, and by comparing the results with those obtained in laboratory before launch. In the following, the cases of AVHRR channels 1 and 2 and of the VISSR/VAS visible channels are discussed separately.

\section{a. AVHRR channels 1 and 2}

Fig. 24 depicts the evolution with time of the AVHRR channels 1 and 2 calibration coefficients. In the figure, the error bars associated to individual points represent the differences due to spatial variations in the White Sands counts. Clearly, there is no evidence of a systematic drift in any of the computed coefficients and no dramatic change occured during the 15-month period of the observations. The gains fluctuate somewhat erratically throughout the study period, by 11 and $15 \%(1 \sigma)$ of the mean values for channels 1 and 2 , respectively. The larger excursion of channel 2 gain likely results from the higher sensitivity of this channel to water vapor (the effect of uncertainties on the water vapor amount is increased). These fluctuations, however, do not appear to be significant at the level of accuracy of our calibration; the errors in the modeled irradiances may easily account for the observed variability.

It is interesting to note that the gains are highly correlated to the viewing zenith angle. The correlation coefficients are -0.77 (channel 1) and -0.83 (channel 2), significant at the $99 \%$ confidence level. This intriguing feature cannot be attributed to errors in the parameterization of gaseous absorption, or even to the fact that absorption-scattering interactions were neglected, because absorption processes act quite differently in the two channels, which would not produce similar correlation coefficients. Also, when examining the model errors and their dependence on the viewing geometry (Figs. 9 and 10), there is no evidence that an increase in viewing zenith angle would be reflected in a decrease of the 


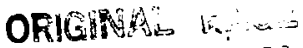

OF POOR Quishiry
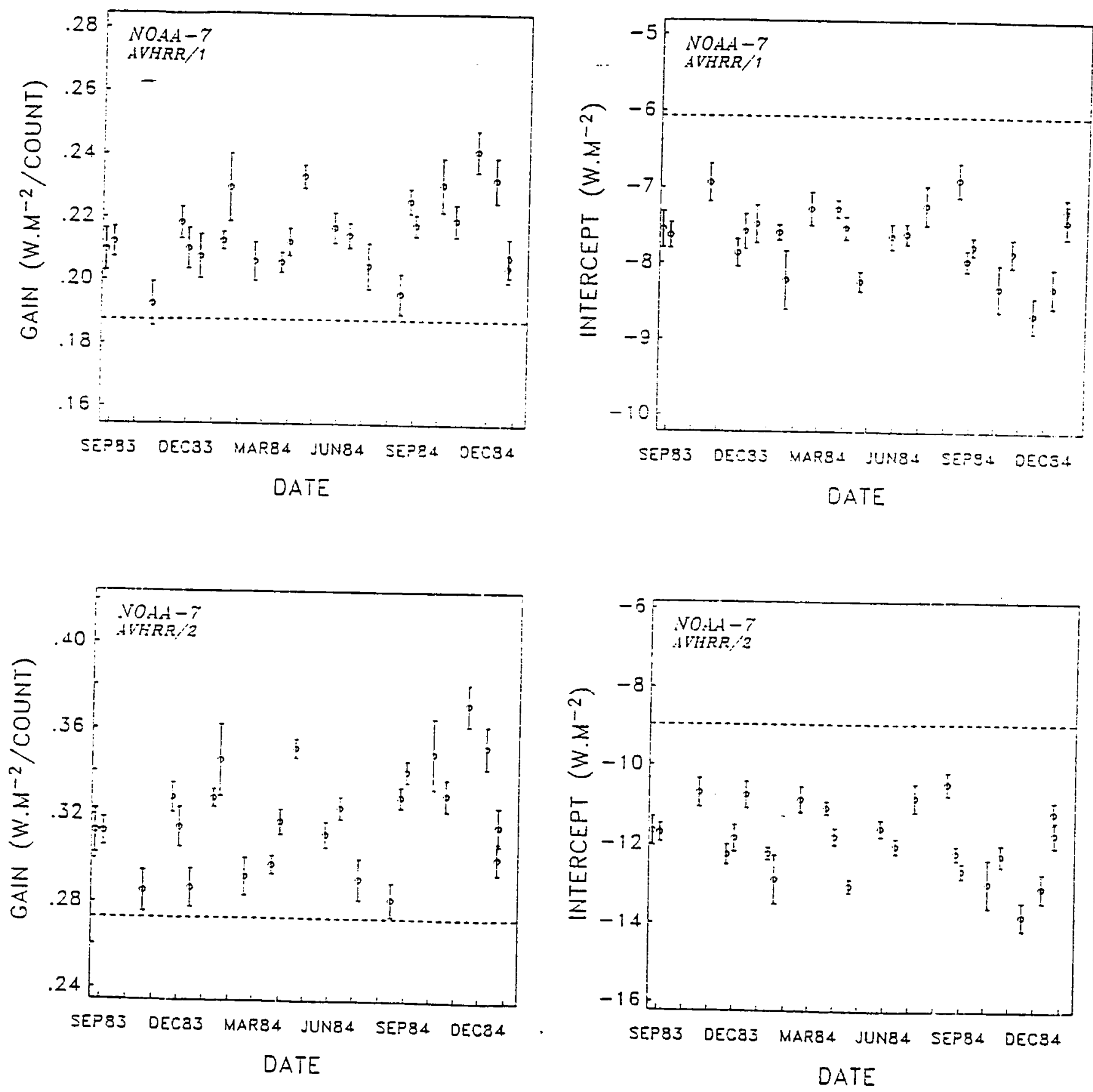

Fig. 24. Time series of AVHRR calibration coefficients determined in orbit. Pre-launch values are represented by dashed lines. 
gains. On the contrary, one would conclude that $\rho^{*}$ is slighty more overestimated at higher viewing zenith angles, yielding a positive correlation. One possible explanation is linked to surface reflectance inhomogeneities. In fact, shading effects are enhanced when the sun is low (recall that $\theta>50^{\circ}$ for most conditions), which reduces the apparent target reflectance at small viewing zenith angles because incidence and observation directions have similar azimuth angles. In other words, $\rho^{*}$ is more overestimated as $\theta$ decreases because more shaded areas occupy the instrument field of view; these areas are implicitly assumed to be illuminated by the sun in the model. The effect is almost impossible to quantify, however, because this would require precise knowledge of the shape and geometry of the dunes and other spatial inhomogeneities; but it might realistically explain the irregular variations of the gains observed in Fig. 24.

When compared to prelaunch gains, the average gains obtained in the present study read higher by about $14 \%$ for channel 1 and $15 \%$ for channel 2 (Fig. 24). The differences are significant, and cannot be explained by model errors: these errors do not contain biases of such magnitudes. In fact, uncertainties in the atmospheric visibility likely introduce a negative bias (see section 5), which would aggravate the disparities. Hovis (1982) pointed out several problems in the technique applied by ITT Aerospace to calibrate the AVHRR solar channels, from the standpoint of the calibration in the laboratory with a sphere and from the fact that there still is no agreement on the value of the solar irradiance in the bands of interest. We used Neckel and Labs' (1984) solar data instead of Thekaekara's (1974) tables, but this cannot quantitatively explain the discrepancies. A likely possibility is therefore that a degradation of the solar channels happened after launch. Since Fig. 24 indicates the occurrence of little or no drift, the solar channels might have deteriorated suddenly. It would certainly be worthwhile to check whether a degratation took place as a result of launch constraints.

So far we have only analyzed changes in the channels 1 and 2 gains. Comparison of intercepts determined in orbit with those before launch (Fig. 24) show that the post-launch values are lower by $2 \mathrm{Wm}^{-2}$ (channel 1) and $3 \mathrm{Wm}^{-2}$ (channel 2). The time variations reflect those of the gains, but naturally are inversely correlated since two-point calibrations were performed. We recall here that the zero offsets (in counts) were found practically constant for the two channels, which is evidence of the stability of the system. 


\section{b. VISSR/VAS visible channels}

In order to assess the stability of the VISSR/VAS solar channels with time, average calibration coefficients were computed for each selected day of the study period. For a given day, two-point calibrations were performed with the observation of space and each of the three White Sands observations, and the results were averaged to provide the daily value. Fig. 25 shows the evolution with time of the gains and intercepts obtained in that way. The error bars in the figure represent the variability of the coefficients during the one-and-one-half period of the observations. The gain changes, about $12 \%$ (GOES-5) and $15 \%$ (GOES-6) of the mean values, are not erratic as those of AVHRR channels 1 and 2. Still, they remain within the uncertainty domain of our calibration (e.g., model errors, transfer function uncertainties and the limitations due to the 6-bit digitization). We note, however, that the VISSR/VAS viewing zenith angles, unlike the AVHRR ones, did not vary much throughout the study period, except for GOES-6 immediately after the failure of GOES-5 (i.e., when GOES-6 was maneuvered to its new position). The solar zenith angle, on the contrary, varied noticeably and a correlation coefficient of 0.66 , significant at the $99 \%$ confidence level, is computed between the GOES- 6 gain and the solar zenith angle. In fact, at fixed or minimally varying viewing zenith angles, $\rho^{*}$ and, consequently, the gains are more overestimated as $\theta_{0}$ increases because, here again, the instruments view more shaded areas. This might be the cause of at least part of the variability in the GOES6 gain. Such a high correlation, however, is not observed for GOES-5. On the contrary, from March 1984 until the failure of the satellite, period during which $\theta_{0}$ decreases, an increase in gain is noted (Fig. 25). This reversed trend might therefore be associated to changes in the system that eventually lead to its collapse.

No systematic effect of the normalization procedure can be detected. However, an abrupt increase in the GOES-5 gain (i.e., a loss of sensitivity) of about $17 \%$ of the mean value is observed between February 2 and February 18, 1984, which cannot be explained by shading effects or inhomogeneities in the surface reflectance. The fact that on March 3 the gain recovers its value of February 18 might be the manifestation of the normalization procedure or other intervention at the ground station. Note that the phenomenon occurred just before the solar channels began to lose sensitivity, which might therefore indicate that something drastic and irreversible happened to the system in orbit at that time. Important variations are also observed during some days, for instance on October 4, 1983 for GOES-5 


\section{ORIGINAL RAUE IS \\ OF POOR QUALTY}
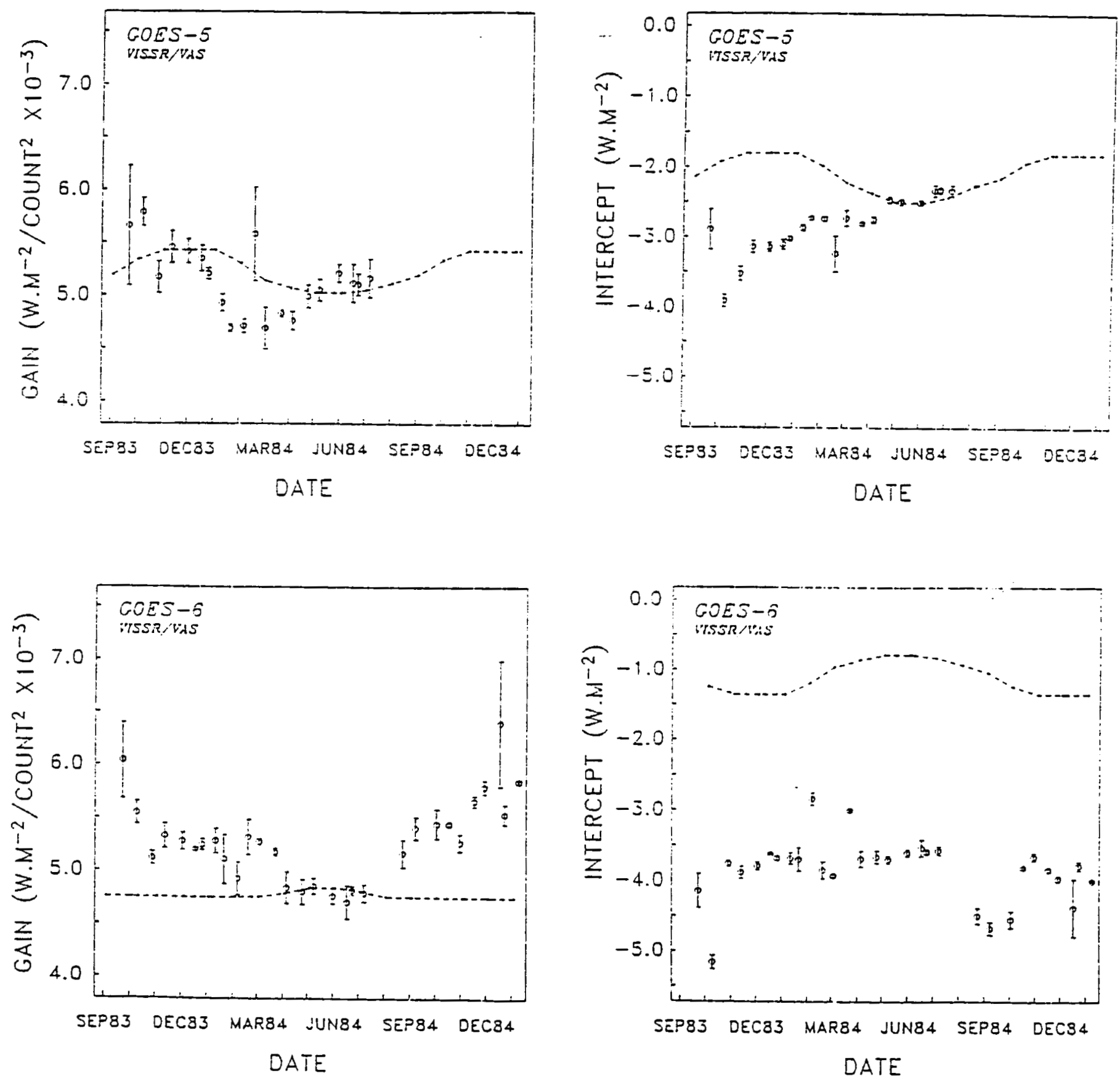

Fig. 25. Same as Fig. 24, but VISSR/VAS instead of AVHRR. 
and December 30, 1984 for GOES-6, and correspond to those cases for which the White Sands brightness counts considerably vary over a half-hour interval. Because the changes do not last, they cannot originate from the normalization procedure. A possible explanation is an error at the ground station that is immediately corrected for.

From the pre-launch calibration curves provided by the Santa Barbara Research Center and the actual scanner temperatures communicated by the NOAA GOES support branch, the pre-launch gains and intercepts were computed and are displayed in Fig. 25 against the post-launch values. The scanner temperatures consequently vary from about $31^{\circ}$ in winter to $10^{\circ}$ in summer, which translates in a significant increase in the sensitivity of the GOES-5 detectors $(8 \%)$ and a slight decrease $(<1 \%)$ in that of the GOES-6 detectors. Deviations of up to $4 \%$ may exist, however, from these average figures between certain detectors. The seasonal changes in the heating effect are not retrieved at all in the postlaunch values of Fig. 25; they are probably masked by the more important variations in the apparent reflectance discussed above. The thermal effect on the intercepts is obviously not visible. These, in other respects, depart from the pre-launch values by $-1 \mathrm{Wm}^{-2}$ (GOES5) and $-3 \mathrm{Wm}^{-2}$ (GOES-6), which generally represent a negligible contribution to the signal measured by the instruments. In a word, Our analysis thus indicates a fairly good agreement between pre- and post-launch calibration coefficients, the discrepancies being largely attributed to spatial inhomogeneities in the surface reflectance, except for GOES-5 from March 1984 until the failure of the satellite period during which the solar channels plausibly deteriorated.

\section{Summary and Recommendations}

The potential of measurements from space of the solar radiation reflected from the earth-atmosphere system for environmental applications has been extensively illustrated in the recent literature. Vegetation index, ground and cloud albedoes, surface insolation and radiation budget at the top of the atmosphere represent a non-exhaustive list of parameters that are discussed in terms of satellite observations in the visible portion of the solar spectrum. Most of the satellite instruments to be used in determining these parameters, unfortunately, are incompletely characterized and calibrated, except those on the Earth Budget Radiation Experiment. Such is the case of the VISSR/VAS and AVHRR radiometers onboard the meteorological satellites operated by NOAA, which do not have active inflight calibration capabilities for the solar channels. 
A simple, probably least expensive, way to routinely calibrate the solar channels of these instruments while they operate in orbit is to use areas on the earth surface as calibration targets. This technique was applied in the present study to the AVHRR onboard NOAA-7 and to the VISSR/VAS onboard GOES-5 and GOES-6. The White Sands Monument area in New Mexico, of well-known bidirectional reflectance, was used as reference standard. Observations taken when the sensors were pointing the deep space served to fix the offset points on the count-versus-irradiance curves. The satellite level irradiances were computed from the ground target characteristics and the optically acting parameters of the overlying atmosphere using a fairly accurate radiative transfer model, which accounts for multiple scattering and bidirectional effects. Atmospheric properties were determined either from climatological data (ozone amount, aerosol phase function) or from observations at the nearest meteorological site (water vapor amount, visibility). The calibration accuracy is believed to be on the order of 8 to $13 \%$, depending on the viewing geometry, surface and atmospheric conditions and on the channel considered.

The results obtained for the 15-month period analyzed indicate the occurence of no significant drift in the solar channels of both types of instrument. The gain changes (about $15 \%$ of the mean values) are largely attributed to spatial inhomogeneities of the calibration site (shading effects due to the presence of dunes) that are not accounted for in the modeling. There is some evidence, however, that the GOES-5 solar channels gradually deteriorated from March 1984 until the failure of the satellite in July 1984. Comparisons of gains determined in orbit with those before launch show that the AVHRR solar channels read higher by about $15 \%$ on the average. This apparent loss of sensitivity cannot be explained by model errors, whose bias component tends to aggravate the discrepancies.

The accuracy of the calibration could certainly be improved by measuring in situ and at the moment of the satellite overpass all the necessary parameters. This would require, however, increased means and effort. In other respects, the non-negligible uncertainties introduced by the inhomogeneity of the target might be avoided by performing the calibration in the flat portion of the White Sands area. A detailed validation of the radiative transfer model is also necessary. In order to do this, a complete data set on atmospheric properties and surface reflectance (including radiation measurements) has been recently acquired for the Yuma desert in Arizona. The Yuma desert, in fact, appears to be a near-homogeneous, very suitable site for the calibration. Its use with White Sands would allow a better verification of the linearity of the detectors with respect to incident energy, 
and more confidence would be placed in the results. In any case, the calibration of the GOES VISSR/VAS and NOAA AVHRR solar channels should be adressed in the context of a global and concerted strategy (e.g., see IAMAP, 1981; WCP, 1986). In view of the requirements for many applications (in particular climate studies), the only way to ensure that the observed variability in the satellite-derived parameters is the result of changes on earth and not in the instruments is to calibrate the data acquired in operations on a continuous basis. As demonstrated in the present study, this can be achieved with our simple and relatively inexpensive method. Nevertheless, episodic (i.e., one or twice during the life of individual satellites) but intensive process-oriented campaigns that involve the utilization of high-flying aircraft should be conducted, even if they are onerous, for they constitute the optimal way of assessing the data quality. 


\section{ACKNOWLEDGEMENTS}

The authors are indebted to D. Tanré of the University of Lille, France, who made available his radiative transfer model. The programming support of $\mathrm{B}$. DiJulio and R. Wylie is gratefully acknowledged, and the authors thank R. Markworth for typesetting the manuscript. The work has benefited from discussions with $\mathbf{R}$. Hoelter of the Santa Barbara Research Center, P. Slater of the University of Arizona, D. Forness of the NOAA GOES support branch and C. Whitlock of the NASA Langley Research Center. The research effort was supported by NASA under contract NAGW-697 and by the California Space Institute. 


\section{REFERENCES}

Brooks, D.R., C.F. England, G.E. Hunt and P. Minnis, 1984: "An Intercalibration of METEOSAT 1 and GOES-2 Visible and Infrared Measurements," J. Atmos. Ocean. Tech., 1, 283-287.

Coulson, K.L. and H. Jacobowitz, 1972: "Proposed Calibration Target for the Visible Channel of a Satellite Radiometer," NOAA/NESS Tech. Rep. \#62.

Deschamps, P.Y., M. Herman and D. Tanré, 1983: "Modélisation du Rayonnement Réfléchi par l'Atmosphère et la Terre, entre 0.35 et $4 \mu \mathrm{m}$," Final Report, ESA Contract $4393 / 80 / \mathrm{F} / \mathrm{DD}(\mathrm{SC})$.

Gautier, C., 1982: “Mesoscale Insolation Variability Derived from Satellite Data," J. Appl. Meteor., 21, 51-58.

Gautier, C., 1986: "Evolution of the Net Surface Shortwave Radiation over the Indian Ocean during Summer MONEX (1979): A Satellite Description," Mon. Wea. Rev., 114, 525-533.

Goody, R.M., 1964: "Atmospheric Radiation," Oxford Univ. Press, New York.

Hovis, W.A. Jr., 1966: "Infrared Spectral Reflectance of Some Common Minerals," Appl. Opt., 5, 245-248.

IAMAP 18, 1981: "Report of Proceedings," III Scientific Assembly, Hamburg, August.

Hovis, W.A., 1982: "Calibration of the NOAA-7 AVHRR/2 Shortwavelength Channel," NOAA/NESS Tech. Memo. E/RA2:WAH.

Hovis, W.A., Jr., J.S. Knoll and G.R. Smith, 1985: "Aircraft Measurements for Calibration of an Orbiting Spacecraft Sensor, ${ }^{, A p p l . ~ O p t ., ~ 24, ~ 407-410 . ~}$

Koepke, P., 1982: "Vicarious Satellite Calibration in the Solar Spectral Range by Means of Calculated Radiances and Its Application to METEOSAT," Appl. Opt., 21, 28452855.

Kriebel, K.I., 1981: "Calibration of the METEOSAT-VIS-Channel by Airborne Measurements," Appl. Opt., 20, 11-12.

Lauritson, L., G.L. Nelson and F.W. Porto, 1979: "Data Extraction and Calibration of TIROS/NOAA Radiometers," NOAA/NESS Tech. Mem. \#107.

London, J., R.D. Bojkov, S. Oltsuans and J.I. Kelly, 1976: "Atlas of the Global distribution of Total Ozone," NCAR/IN/133+STR.

Malkmus, W., 1967: "Random Lorenz Band Model with Exponential-Tailed S ${ }^{-1}$ LineIntensity Distribution Function," J. Opt. Soc. Am., 57, 323-329.

McClatchey, R.A., R.W. Fenn, J.E.A. Selby, F.E. Voltz and J.S. Garine, 1971: "Optical Properties of the Atmosphere," AFCRL 71-0279, Envir. Res. Paper \#354.

Neckel, H. and D. Labs, 1984: "The Solar Radiation between 3300 and $12500 \AA$," Sol. Phys., 90, 205-258.

Rockwood, A.A. and S.K. Cox, 1978: "Satellite Inferred Surface Albedo over Northwestern Africa," J. Atmos. Sci., 35, 513-522.

Smith, E.A. and T.H. Vonder Haar, 1980: "A First Look at the Summer MONEX GOES Satellite Data," Proc. 15th AIAA Thermophysics Conf., Snowmass, CO, 14-16 July. 
Smith, E.A. and D. Loranger, 1977: "Radiometric Calibration of Polar and Geosynchronous Satellite Shortwave Detectors for Albedo Measurements," Dept. of Atmos. Sciences, Colorado State Univ., Fort Collins (unpublished manuscript).

Staff Members, 1980: "Visible Infrared Spin Scan Radiometer Atmospheric Sounder System Description," Santa Barbara Research Center, Goleta, CA.

Stephens, G.L., G.G. Campbell and T.H. Vonder Haar, 1981: "Earth Radiation Budgets," J. Geophys. Res., 86, 9739-9760.

Thekaekara, M.P., 1974: “Extraterrestrial Solar Spectrum, 3000-61000 Åat $1 \AA$ Intervals," Appl. Opt., 13, 518-522.Tanré, D., M. Herman, P.Y. Deschamps and A. Deleffe, 1979: "Atmospheric Modelling for Space Measurements of Ground Reflectances, Including Bidirectional Properties," Appl. Opt., 18, 3587-3596.

Tanré, D., M. Herman and P.Y. Deschamps, 1983: "Influence of the Atmosphere on Space Measurements of Directional Properties," Appl. Opt., 21, 733-741.

Tanré, D., C. Deroo, P. Duhaut, M. Herman, J.J. Morcrette, J. Perbos and P.Y. Deschamps, 1985: "Effets Atmosphériques en Télédétection-Logiciel de Simulation du Signal Satellitaire dans le Spectre Solaire," Proc. 3rd. Int. Coll. on Spectral Signatures of Objects in Remote Sensing, Les Arcs, France, 16-20 December.

Tarpley, J.D., S.R. Schneider and R.L. Money, 1984: "Global Vegetation Indices from the NOAA-7 Meteorological Satellite," J. Climate Appl. Meteor., 23, 491-494.

Tucker, C.J., J. A. Gatlin and S.R. Schneider, 1984: "Monitoring Vegetation in the Nile Delta with NOAA-6 and NOAA-7 AVHRR Imagery," Photo. Eng. Remote Sens., 50, 53-61.

Walraven, R.L. and K.L. Coulson, 1972: "Measurements of the Light Properties of Gypsum Sand," contrib. Atmos. Sci., No. 7, Univ. of California, Davis.

WCP 55, 1983: "Report of the WMO Radiation Commission of IAMAP Meeting of Experts on Aerosols and their Climatic Effects," Williamsburg, VA, 28-30 March.

WCP 115, 1986: "Report of the Workshop on Surface Radiation Budget for Climate Applications," Columbia, MD, 18-21 June.

Williamson, L.E., 1975: "Satellite Calibration Data," Annual Data Report, 1974, Tech. Rep. ECOM-DR-75-1, Atmos. Sciences Lab., White Sands Missile Range, NM. 